\title{
ON COVERING BY TRANSLATES OF A SET
}

\author{
BÉLA BOLLOBÁS, SVANTE JANSON, AND OLIVER RIORDAN
}

\begin{abstract}
In this paper we study the minimal number $\tau(S, G)$ of translates of an arbitrary subset $S$ of a group $G$ needed to cover the group, and related notions of the efficiency of such coverings. We focus mainly on finite subsets in discrete groups, reviewing the classical results in this area, and generalizing them to a much broader context. For example, the worst-case efficiency when $S$ has $k$ elements is of order $1 / \log k$. We show that if $n(k)$ grows at a suitable rate with $k$, then almost every $k$-subset of any given group with order $n$ comes close to this worstcase bound. In contrast, if $n(k)$ grows very rapidly, or if $k$ is fixed and $n \rightarrow \infty$, then almost every $k$-subset of the cyclic group with order $n$ comes close to the optimal efficiency.
\end{abstract}

\section{INTRODUCTION}

Packing and covering problems have been studied for many decades, both in discrete geometry and in stochastic geometry. One of the basic questions of discrete geometry is the following: given sets $S, T \subset \mathbb{R}^{d}$, what is the minimal number of translates of $S$ that cover $T$ ? The most studied cases are when $S$ is a ball in $\mathbb{R}^{d}$, or a convex polygon in $\mathbb{R}^{2}$. For these and other questions see the classical treatises of Fejes Tóth [17; 18] and Rogers 45]. For a selection of classical and more recent results see, for example, [4; 5 ; 9 ; 13 ; $16 ; 22 ; 41$.

In stochastic geometry similar questions are considered. Here the starting point was the study of the probability that $n$ random arcs, each of length $a$, cover the entire circle; see Steutel [51], Flatto [19] and Janson 25;; 27]. For general random covering problems see the books by Penrose [43], Meester and Roy [37] and Hall [23], and a host of papers including [1; 2; 3; 26; 36; 42].

In almost all cases above, the set $S$ is taken to be convex. In this paper we shall address a rather different kind of covering problem, studying the minimal number of translates of a given, arbitrary subset $S$ of a general group $G$ needed to cover the group. Of course, in this generality not too much can be said, so we shall focus on various natural special cases. Although the general problem includes those concerning balls mentioned above, the flavour of the cases we study turns out to be closer to covering problems in

Date: October 19, 2009; revised May 25, 2010.

The first author's research was supported in part by NSF grants CNS-0721983, CCF0728928 and DMS-0906634, and ARO grant W911NF-06-1-0076. 
stochastic geometry. Some of the special cases we consider have been studied before: for example, Newman [38] studied the density of coverings of $\mathbb{Z}$ by a given finite set (in fact, he studied coverings of the natural numbers, which turns out to be equivalent), and Schmidt [47] proved results about coverings of $\mathbb{Z}^{d}$ (as well as various other cases). We shall comment on the relationship of our results to these earlier results in the relevant sections.

Let $G$ be a group and let $S$ be a non-empty subset of $G$. By the covering number $\tau(S, G)$ we mean the smallest number of (left) translates of $S$ that cover $G$, i.e.,

$$
\begin{aligned}
\tau(S, G) & :=\min \left\{m: \bigcup_{i=1}^{m} t_{i} S=G \text { for some } t_{1}, \ldots, t_{m} \in G\right\} \\
& =\min \{|T|: T S=G\}
\end{aligned}
$$

If no finite set of translates of $S$ covers $G$, then we set $\tau(S, G)=\infty$. Our aim is to study the efficiency of coverings by $S$, loosely defined as the reciprocal of the average number of times an element is covered in a covering achieving the minimum $\tau(S, G)$.

We are mainly interested in Abelian groups, for which we use additive notation, and in particular the groups $\mathbb{Z}_{n}, \mathbb{Z}$ and $\mathbb{R}$, and their powers. In general, however, $G$ can be any group, and need not be Abelian.

In the following sections we consider some natural special cases, describing the appropriate notions of covering multiplicity and covering efficiency, and proving various results. Our main results focus on the worst possible efficiency of sets $S$ in some class, for example the class of $k$-element subsets of $\mathbb{Z}$. We also include many examples and trivial results to illustrate the definitions, and, perhaps most importantly, we pose many open problems.

After giving the definitions for general compact groups in Section 2, we first consider finite groups, presenting our basic results in Section 3, and considering random subsets $S$ in Section 4 . We turn to subsets of $\mathbb{Z}$ in Section 5. In Sections 6 and 7 , we compare coverings of $\mathbb{Z}$ with those of cyclic groups, focusing on small sets $S$. In Sections 810 we consider the case $G=\mathbb{R}$. Finally, in Sections 11 and 12 we consider coverings of $\mathbb{Z}^{d}$ and $\mathbb{R}^{d}$, respectively; these sections contain (essentially) only questions, rather than results.

Acknowledgements. This research was begun during a visit by SJ to the University of Cambridge, partly funded by Trinity College, Cambridge, and continued during a visit by SJ to the Isaac Newton Institute in Cambridge, funded by a Microsoft fellowship. We are grateful to two anonymous referees for careful reading of the paper, for finding two minor errors, and for helpful suggestions concerning the presentation. 


\section{2. $G$ COMPACT}

If $G$ is a compact group, it has a finite Haar measure, which we denote by $\mu$. We consider only the case when $S$ is measurable and $\tau(S, G)<\infty$; note that the latter holds whenever $S$ has an interior point.

When $G$ is compact and $\tau(S, G)<\infty$, we define the covering multiplicity of $S$ as

$$
\kappa(S, G):=\frac{\tau(S, G) \mu(S)}{\mu(G)},
$$

this is the average number of times each point in $G$ is covered by a smallest (minimum cardinality) covering by translates of $S$. The efficiency of $S$ is

$$
\varepsilon(S, G):=1 / \kappa(S, G) .
$$

Note that these definitions do not depend on the chosen normalisation of the Haar measure $\mu$.

If $T S=G$, then $\mu(G)=\mu\left(\bigcup_{t \in T} t S\right) \leq|T| \mu(S)$, so trivially

$$
\tau(S, G) \geq \frac{\mu(G)}{\mu(S)}
$$

and thus

$$
\begin{aligned}
& 1 \leq \kappa(S, G)<\infty, \\
& 0<\varepsilon(S, G) \leq 1 .
\end{aligned}
$$

We also define the covering multiplicity $\kappa(\mathcal{T})$ or efficiency $\varepsilon(\mathcal{T})$ of a particular covering $\mathcal{T}=\left\{t_{i} S\right\}$ of $G$ :

$$
\kappa(\mathcal{T}):=|\mathcal{T}| \mu(S) / \mu(G) \quad \text { and } \quad \varepsilon(\mathcal{T}):=1 / \kappa(\mathcal{T}) .
$$

The analogues of (2.4) and (2.5) hold for these too. Trivially, $\kappa(S, G)=$ $\min \{\kappa(\mathcal{T})\}$, where the minimum is over coverings $\mathcal{T}$ by translates of $S$, and $\varepsilon(S, G)=\max \{\varepsilon(\mathcal{T})\}$.

We call a subset $S$ of $G$ efficient, or efficiently covering, if $\kappa(S, G)=$ $\varepsilon(S, G)=1$. Typically, $\kappa(S, G)>1$ and thus $\varepsilon(S, G)<1$, in which case $S$ is inefficient. Of course, there many efficient sets $S$; here is one simple family of examples.

Example 2.1. If $S$ is a subgroup of $G$ of finite index, then $\tau(S, G)=$ $\mu(G) / \mu(S)$ and $\kappa(S, G)=\varepsilon(S, G)=1$.

In general, the study of efficient coverings, or, essentially equivalently, of tilings, has received much more attention than the inefficient case. One early example is the paper of Hajós [21]; we shall mention some other examples in specific contexts later. Here we focus on the inefficient case, and specifically on the question of how large $\kappa(S, G)$ is, rather than simply whether it is equal to 1 or not. 
Remark 2.2. We may also define the effective size of $S$ as

$$
\nu(S, G):=\frac{\mu(G)}{\tau(S, G)}=\frac{\mu(S)}{\kappa(S, G)}=\varepsilon(S, G) \mu(S),
$$

which satisfies $0<\nu(S, G) \leq \mu(S)$. Thus, as far as coverings are concerned, $S$ is 'as good as' an efficiently covering set of measure $\nu(S, G)$. Analogous definitions with $\nu(S):=\varepsilon(S) \mu(S)$ can be made for the other cases studied in later sections, in particular for $G=\mathbb{Z}$ and $G=\mathbb{R}$. Although the quantity $\nu$ also carries intuitive information, we shall work throughout with $\tau, \kappa$ and $\varepsilon$.

\section{3. $G$ FINITE}

An important special case is when $G$ is finite, in which case we use the counting measure $\mu(A)=|A|$ as the (normalized) Haar measure. Note that now $\tau(S, G) \leq|G|<\infty$ for every non-empty set $S$. Hence, for any nonempty $S \subseteq G$,

$$
\begin{aligned}
\frac{|G|}{|S|} & \leq \tau(S, G) \leq|G|, \\
1 & \leq \kappa(S, G) \leq|S|, \\
|S|^{-1} & \leq \varepsilon(S, G) \leq 1 .
\end{aligned}
$$

The lower bounds on $\tau$ and $\kappa$, or, equivalently, the upper bound $\varepsilon \leq 1$, can be attained, for example in Examples 2.1 and 6.3. Indeed, when $G$ is finite, a covering $\mathcal{T}=\left\{t_{i} S\right\}$ is efficient (i.e., $\varepsilon(\mathcal{T})=1$ ) if and only if it is a partition of $G$ into disjoint translates of $S$. On the other hand, the lower bound on $\varepsilon$ (upper bounds on $\tau$ and $\kappa$ ) can be substantially improved as we shall now see.

One method of finding a covering set of translates is the greedy algorithm. Pick elements $t_{1}, t_{2}, \ldots$ in $G$ one by one as follows: having chosen $t_{1}, \ldots, t_{j-1}$ with $\bigcup_{i=1}^{j-1} t_{i} S \neq G$, choose $t_{j}$ so as to maximize $\left|\bigcup_{i=1}^{j} t_{i} S\right|$. (If several choices of $t_{j}$ achieve the maximum, pick one of them by any rule.) The algorithm terminates when $\bigcup_{i=1}^{j} t_{i} S=G$. We shall write $\tau_{\text {Greedy }}(S, G)$ for the final value of $j$, i.e., the number of translates of $S$ in the covering produced by the greedy algorithm.

Theorem 3.1. Suppose that $G$ is a finite group with $|G|=n$, and let $S \subseteq G$ with $|S|=k \geq 1$. Define integers $n_{j}$ recursively by $n_{0}=n$ and

$$
n_{j+1}=\left\lfloor n_{j}(1-k / n)\right\rfloor, \quad j \geq 1 .
$$

Then

$$
\tau(S, G) \leq \tau_{\text {Greedy }}(S, G) \leq \min \left\{j: n_{j}=0\right\} .
$$

Proof. Let $m_{j}:=\left|G \backslash \bigcup_{i=1}^{j} t_{i} S\right|$ be the number of uncovered elements after $j$ steps of the greedy algorithm. Since a random choice of $t_{j+1}$ covers on 
average $m_{j}|S| /|G|$ of these, the optimal choice covers at least as many and thus

$$
m_{j+1} \leq m_{j}-m_{j}|S| /|G|=m_{j}(1-k / n) .
$$

Since $m_{j+1}$ is an integer we have $m_{j+1} \leq\left\lfloor m_{j}(1-k / n)\right\rfloor$, and so, by induction, $m_{j} \leq n_{j}$ for every $j \geq 0$. In particular, if $n_{j}=0$ then $m_{j}=0$ and thus $\bigcup_{i=1}^{j} t_{i} S=G$, which yields (3.5).

The simplest way to obtain an explicit bound on $\tau(S, G)$ from Theorem 3.1 is to observe that (3.4) implies $n_{j} \leq n(1-k / n)^{j}<n e^{-j k / n}$. Setting $\ell=$ $\frac{n}{k} \log n$, this gives $n_{\lceil\ell\rceil}<1$, so $n_{\lceil\ell\rceil}=0$ and $\tau(S, G) \leq\lceil\ell\rceil$. In fact, we can do better.

Let $H_{k}:=\sum_{j=1}^{k} 1 / j$ denote the $k$ th harmonic number. It is well known that $\log k<H_{k} \leq \log k+1$.

Corollary 3.2. Let $G, S, n, k$ be as in Theorem 3.1. Then

$$
\begin{aligned}
\tau(S, G) & \leq \frac{n}{k} H_{k} \leq \frac{n}{k}(\log k+1), \\
\kappa(S, G) & \leq H_{k} \leq \log k+1, \\
\varepsilon(S, G) & \geq \frac{1}{H_{k}} \geq \frac{1}{\log k+1} .
\end{aligned}
$$

Proof. Let $N(t)$ be defined for $t \geq 0$ by $N(j)=n_{j}$ for integer $j$ and by linear interpolation between integers. Then $N(t)$ is continuous and nonincreasing, with $N(0)=n$ and $N(t)=0$ for large $t$. For $0 \leq i \leq k$, let $t_{i}:=\min \{t: k N(t) / n \leq i\}$; thus $0=t_{k}<t_{k-1}<\cdots<t_{0}$.

If $t>0$ is not an integer, then, setting $j:=\lfloor t\rfloor$, so $j<t<j+1$, we have

$$
-N^{\prime}(t)=n_{j}-n_{j+1}=\left\lceil n_{j} k / n\right\rceil \geq\lceil k N(t) / n\rceil .
$$

If $t_{i}<t<t_{i-1}$, then $k N(t) / n>i-1$ and thus $-N^{\prime}(t) \geq i$. Hence,

$$
i\left(t_{i-1}-t_{i}\right) \leq \int_{t_{i}}^{t_{i-1}}-N^{\prime}(t) d t=N\left(t_{i}\right)-N\left(t_{i-1}\right)=\frac{n}{k} i-\frac{n}{k}(i-1)=\frac{n}{k} .
$$

Consequently, using (3.5) and noting that $t_{0}=\min \{t: N(t)=0\}$ is an integer by the definition of $N$,

$$
\tau(S, G) \leq \tau_{\text {Greedy }}(S, G) \leq t_{0}=\sum_{i=1}^{k}\left(t_{i-1}-t_{i}\right) \leq \sum_{i=1}^{k} \frac{n}{k i}=\frac{n}{k} H_{k} .
$$

Remark 3.3. Lorentz [35] applied the greedy algorithm in a slightly different context (coverings of the natural numbers by translates of an infinite set); he remarked that his method applies (in our terminology) to bound $\tau\left(S, \mathbb{Z}_{n}\right)$ when $|S|=k$, although he only stated the weak bound $O(n \log k / k)$ for this case.

Newman [38] proved a result related to Corollary 3.2 but for coverings of the natural numbers, or equivalently of $\mathbb{Z}$ : he showed that if $S \subset \mathbb{Z}$ with $|S|=k$, then $\kappa(S, \mathbb{Z})$ (which we define in Section 5 ) satisfies $\kappa(S, \mathbb{Z}) \leq \log k+$ 1. His method (picking translates randomly to cover most of $G$ and then 
using one translate for each remaining element) applies just as well to subsets of a finite group, but gives the slightly worse bound $\kappa(S, G) \leq \min \{k s / n+$ $k \exp (-k s / n)\}$ (corresponding to $\tau(S, G) \leq \min \{s+n \exp (-k s / n)\}$ ), where the minimum is over integer $s$. Note that the minimum over real $s$ is $\log k+1$, attained at $s=(n / k) \log k$.

Remark 3.4. There is an interesting connection between the covering problem considered here, and the algorithmic problem SET COVER; we are grateful to a referee for bringing this to our attention. In the SET COVER problem, the input is a family $\mathcal{S}=\left\{S_{i}\right\}$ of sets covering some ground set $X$ (i.e., with union $X$ ), and the task is to find efficiently a subcover of close to minimum size. Johnson [30] showed that the greedy algorithm (pick sets one by one, choosing one that includes the maximum number of uncovered points) achieves an approximation ratio of at most $H_{k}$ when $\left|S_{i}\right| \leq k$ for all $i$. The proof is very different from that above: he considers the weight function $w(U)=\sum_{S_{i} \in \mathcal{F}} H_{\left|S_{i} \cap U\right|}$, where $\mathcal{F} \subseteq \mathcal{S}$ is a family covering $X$ with $|\mathcal{F}|$ minimal. Let $U \subseteq X$ denote the set of points as yet uncovered at some stage. If the greedy algorithm next chooses a set $S_{j}$ covering $r$ points of $U$, then $\left|S_{i} \cap U\right| \leq r$ for all $S_{i} \in \mathcal{F}$. Each newly covered point decreases $\left|S_{i} \cap U\right|$ by one for (at least) one $S_{i} \in \mathcal{F}$, so the weight function of the uncovered points decreases by at least $r \min \left\{H_{a}-H_{a-1}: a \leq r\right\}=r / r=1$. (Several different points may decrease the same $\left|S_{i} \cap U\right|$, but this is no problem.) Hence the algorithm uses at most $w(X)=\sum_{i} H_{\left|S_{i}\right|} \leq H_{k}|\mathcal{F}|$ sets.

A simple modification of this argument gives an alternative proof of Corollary 3.2. Suppose $\mathcal{S}$ is a cover of $X$, and $\mathcal{F} \subseteq \mathcal{S}$ is an $\ell$-cover of $X$, so each $x \in X$ is in at least $\ell$ sets in $\mathcal{S}$. Taking $w(U)=\sum_{S_{i} \in \mathcal{F}} H_{\left|S_{i} \cap U\right|}$ as before, when running the greedy algorithm to find a (1-)cover of $X$, the weight of the uncovered points decreases by at least $\ell$ at every step, since each newly covered point is in at least $\ell$ sets $S_{i} \in \mathcal{F}$. If all $S_{i}$ have size at most $k$, the greedy algorithm thus uses at most $w(X) / \ell=H_{k}|\mathcal{F}| / \ell$ sets. Taking $X=G$, $\mathcal{S}=\{t+S: t \in G\}, \ell=k=|S|$ and $\mathcal{F}=\mathcal{S}$, this gives (3.6).

Remark 3.5. Together, (3.4) and (3.5) give the best bounds obtainable by the simple argument in the proof of Theorem 3.1. However, this implicit form is not very useful, which is why we give the explicit (inexact) estimates for $n_{j}$ above. Other estimates of $n_{j}$ yield other estimates of $\tau(S, G)$.

For example, noting that $n_{j} \leq n(1-k / n)^{j}$, if

$$
j=\lfloor\log n /|\log (1-k / n)|\rfloor+1>\log n /|\log (1-k / n)|,
$$

then $n_{j}<1$ and thus $n_{j}=0$. Hence, (3.5) implies

$$
\tau(S, G) \leq \frac{\log n}{|\log (1-k / n)|}+1 .
$$

Alternatively, taking $j=\lceil\log k /|\log (1-k / n)|\rceil$, we similarly obtain

$$
n_{j} \leq n(1-k / n)^{j} \leq n / k ;
$$


thus at most $n / k$ further steps are needed and $\tau_{\text {Greedy }}(S, G) \leq j+n / k$, which yields

$$
\tau(S, G) \leq \frac{\log k}{|\log (1-k / n)|}+\frac{n}{k}+1 .
$$

If $k$ is comparable with $n$ (for example, $k=n / 2$ ), then (3.9) and (3.10) are better than (3.6). However, we are usually interested in $n$ much larger than $k$, or even $n \rightarrow \infty$ with $k$ fixed. Then (3.6) and (3.10) give similar bounds (with the former much cleaner), and (3.9) is much weaker.

Returning to trivialities, we next note that one can bound the efficiency of the product of two sets in terms of those of the sets themselves.

Lemma 3.6. Let $S_{1} \subseteq G_{1}$ and $S_{2} \subseteq G_{2}$, where $G_{1}$ and $G_{2}$ are finite groups. Then

$$
\varepsilon\left(S_{1}, G_{1}\right) \varepsilon\left(S_{2}, G_{2}\right) \leq \varepsilon\left(S_{1} \times S_{2}, G_{1} \times G_{2}\right) \leq \min \left\{\varepsilon\left(S_{1}, G_{1}\right), \varepsilon\left(S_{2}, G_{2}\right)\right\}
$$

or, equivalently,

$$
\kappa\left(S_{1}, G_{1}\right) \kappa\left(S_{2}, G_{2}\right) \geq \kappa\left(S_{1} \times S_{2}, G_{1} \times G_{2}\right) \geq \max \left\{\kappa\left(S_{1}, G_{1}\right), \kappa\left(S_{2}, G_{2}\right)\right\} .
$$

Proof. It is more convenient to prove the second form (3.11). For the first inequality, we simply take the product of two coverings: choose $T_{i} \subseteq G_{i}$ with $T_{i} S_{i}=G_{i}$ and $\left|T_{i}\right|=\tau\left(S_{i}, G_{i}\right)$. Then $\left(T_{1} \times T_{2}\right)\left(S_{1} \times S_{2}\right)=\left(T_{1} S_{1}\right) \times\left(T_{2} S_{2}\right)=$ $G_{1} \times G_{2}$, so $\tau\left(S_{1} \times S_{2}, G_{1} \times G_{2}\right) \leq\left|T_{1} \times T_{2}\right|=\tau\left(S_{1}, G_{1}\right) \tau\left(S_{2}, G_{2}\right)$, which gives the inequality.

For the second inequality, consider any covering of $G_{1} \times G_{2}$ by translates of $S_{1} \times S_{2}$. For each $g \in G_{1}$, this induces a covering of the copy $\{g\} \times G_{2}$ of $G_{2}$ by translates of $S_{2}$. By definition of $\kappa\left(S_{2}, G_{2}\right)$, each element is covered on average at least $\kappa\left(S_{2}, G_{2}\right)$ times. Averaging over $g$, we see that in the covering of $G_{1} \times G_{2}$, each element is on average covered at least $\kappa\left(S_{2}, G_{2}\right)$ times. Hence $\kappa\left(S_{1} \times S_{2}, G_{1} \times G_{2}\right) \geq \kappa\left(S_{2}, G_{2}\right)$. Similarly $\kappa\left(S_{1} \times S_{2}, G_{1} \times\right.$ $\left.G_{2}\right) \geq \kappa\left(S_{1}, G_{1}\right)$.

Of course, Lemma 3.6 applies just as well in the compact setting, with suitable modifications to the proof. Unsurprisingly, the inequalities may be strict. For example, let $G_{1}=G_{2}$ be the cyclic group with 5 elements and let $S_{1}=S_{2}$ be a subset of $G_{1}$ of size 4 . It is easy to see that $\tau\left(S_{i}, G_{i}\right)=2$, while $\tau\left(S_{1} \times S_{2}, G_{1} \times G_{2}\right)=3$. This gives $\kappa\left(S_{i}, G_{i}\right)=8 / 5$ and $\kappa\left(S_{1} \times S_{2}, G_{1} \times G_{2}\right)=$ 48/25. We shall give a less trivial example where the first inequality in (3.11) is strict in Example 4.8 .

\section{RANDOM $S$ IN A Finite GRoup}

In this section we shall show that the bounds in Corollary 3.2 are close to best possible, in that the worst case efficiency for sets $S$ of size $k$ really is as small as $(1+o(1)) / \log k$. Newman [38] proved such a result for subsets

of the natural numbers (or, equivalently, of $\mathbb{Z}$, or of $\mathbb{Z}_{n}$ with $n$ large), using 
a random construction; although his argument adapts easily to our setting, we shall give a different proof that seems to generalize more easily.

In fact, we shall show a little more: if $n=n(k)$ grows at a suitable rate, with $n / k$ tending to infinity but not too fast, then almost any $k$-element subset of any given $n$-element group covers inefficiently. This contrasts with our results in Section 17, where we show that in the cyclic case, if $n$ grows very rapidly with $k$, then almost all $k$-element subsets cover with close to optimal efficiency.

Theorem 4.1. Given $0<\delta<1$, for all sufficiently large $k$ there exists a finite group $G$ and a subset $S$ of size $k$ with $\kappa(S, G) \geq(1-\delta) \log k$. In particular, $G$ can be chosen as any group of order $\lfloor k \log k\rfloor$, for example a cyclic group.

Proof. Pick positive integers $n=n(k)$ and $t=t(k)$ so that $n / k \rightarrow \infty$,

$$
t k / n \sim(1-2 \delta / 3) \log k
$$

as $k \rightarrow \infty$, and $t \leq k^{\delta / 6}$ for sufficiently large $k$. For example, we may take $n=\lfloor k \log k\rfloor$ and $t=\left\lfloor(1-2 \delta / 3)(\log k)^{2}\right\rfloor$.

Let $G$ be any group of order $n$. Set $p=k / n=o(1)$, and let $S$ be a random subset of $G$ obtained by selecting each element independently with probability $p$, so $|S|$ has a binomial distribution with mean $k$.

We shall show that for any $T \subset G$ with $|T|=t$, the probability that $T S=$ $G$ is $o\left(1 /\left(\begin{array}{l}n \\ t\end{array}\right)\right)$ as $k \rightarrow \infty$, uniformly in $T$. It follows that the expected number of 'good' sets $T$ for a given $S$ is $o(1)$, so with probability $1-o(1)$, there is no good set for $S$. Since $\mathbb{P}(|S| \geq k)>1 / 2$, for $k$ large enough it follows that with positive probability, $|S| \geq k$ and $\tau(S, G)>t$. In particular, deleting some elements if necessary, there is some $S$ with $|S|=k$ and $\tau(S, G)>t$, and the result follows.

To carry out this plan, fix a set $T \subset G$ with $|T|=t$. For $y \in G$, let $I_{y}$ denote the indicator function of the event that $y$ is not in $S$, so $\mathbb{E}\left(I_{y}\right)=1-p$. For $x \in G$, let $U_{x}$ denote the indicator function of the event that $x$ is not covered by $T S$. Then

$$
U_{x}=\prod_{z \in T} I_{z^{-1} x}
$$

so $\mathbb{E}\left(U_{x}\right)=(1-p)^{t}$. Finally, let $N=\sum_{x} U_{x}$ be the number of uncovered points. Then, for $k$ large enough,

$$
\begin{aligned}
\mu:=\mathbb{E}(N)=n(1-p)^{t}=n & \exp (-(1-o(1)) t k / n) \\
& \geq n \exp (-(1-\delta / 2) \log k)=k^{\delta / 2}(n / k) .
\end{aligned}
$$

Let us write $x \sim y$ if the indicator functions $U_{x}$ and $U_{y}$ are dependent. From (4.2), we have $x \sim y$ if and only if there are $z, z^{\prime} \in T$ such that $z^{-1} x=\left(z^{\prime}\right)^{-1} y$, i.e., such that $z^{\prime} z^{-1}=y x^{-1}$. In other words $x \sim y$ if and only if $y x^{-1} \in T T^{-1}=\left\{z^{\prime} z^{-1}: z, z^{\prime} \in T\right\}$. Hence, for each $x$, the number 
of $y$ such that $x \sim y$ is $\left|T T^{-1}\right| \leq t^{2}$. Let

$$
\bar{\Delta}=\sum_{x \sim y} \mathbb{E}\left(U_{x} U_{y}\right) \leq \sum_{x \sim y} \mathbb{E}\left(U_{x}\right) \leq t^{2} \sum_{x} \mathbb{E}\left(U_{x}\right)=t^{2} \mu,
$$

where the sum runs over all ordered pairs $x \sim y$, including those with $x=y$. By Janson's inequality [28, Theorem 2.18],

$$
\mathbb{P}(T S=G)=\mathbb{P}(N=0) \leq \exp \left(-\frac{\mu^{2}}{\bar{\Delta}}\right) \leq \exp \left(-\frac{\mu^{2}}{t^{2} \mu}\right)=\exp \left(-\mu / t^{2}\right) .
$$

Recalling that $t \leq k^{\delta / 6}$, from (4.3) we have $\mu / t^{2} \geq k^{\delta / 6}(n / k)$. For $k$ sufficiently large we have

$$
\left(\begin{array}{l}
n \\
t
\end{array}\right) \leq\left(\frac{e n}{t}\right)^{t} \leq k^{t}=\exp (t \log k) \leq \exp \left(\frac{n}{k}(\log k)^{2}\right) .
$$

For large $k,(\log k)^{2}$ is much smaller than $k^{\delta / 6}$, and it follows that

$$
\sum_{|T|=t} \mathbb{P}(T S=G) \leq\left(\begin{array}{c}
n \\
t
\end{array}\right) \exp \left(-\frac{\mu}{t^{2}}\right) \leq \exp \left(\frac{n}{k}(\log k)^{2}-\frac{n}{k} k^{\delta / 6}\right)=o(1),
$$

and the result follows.

Remark 4.2. The proof above shows that for $n(k)$ in a certain range, namely $n=k^{1+o(1)}$ with $n / k \rightarrow \infty$, almost all $k$-elements subsets $S$ of any group of order $n$ have $\kappa(S, G) \geq(1-\delta) \log k$, in the sense that the proportion of such sets tends to 1 as $k \rightarrow \infty$. (See Theorem 4.7 below for an even stronger result.) As written, the argument extends to larger $n(k)$ only if we are willing to accept a decrease in the claimed inefficiency. However, we were rather careless in the proof, simply estimating $\mathbb{E}\left(U_{x} U_{y}\right)$ by $\mathbb{E}\left(U_{x}\right)$. Indeed, one in fact has

$$
\mathbb{E}\left(U_{x} U_{y}\right)=(1-p)^{\left|T^{-1} x \cup T^{-1} y\right|}=(1-p)^{2 t-\left|T^{-1} x \cap T^{-1} y\right|}=(1-p)^{2 t-r\left(y x^{-1}\right)},
$$

where $r\left(y x^{-1}\right)$ is the number of ways of writing $y x^{-1}$ as $z^{\prime} z^{-1}$ with $z^{\prime}$, $z \in T$. We have effectively bounded $r\left(y x^{-1}\right)$ by $t$ for all $y x^{-1}$, whereas one could consider how often $r\left(y x^{-1}\right)$ can be large. In doing so, one may have to consider special sets $T$, such as (in the Abelian case) those containing large arithmetic progressions, separately: one needs the sum over $T$ of the probability that $T S=G$ to be small, rather than a uniform bound for each $T$.

Note that there is a limit to how rapidly $n$ can be allowed to grow with $k$; we shall show in Section 7 that if $n$ grows very rapidly with $k$, or if $k$ is fixed and $n \rightarrow \infty$, then in the cyclic group $\mathbb{Z}_{n}$, for any $\delta>0$ almost all $k$-element subsets have $\kappa\left(S, \mathbb{Z}_{n}\right) \leq 1+\delta$. In general, it seems to be an interesting (if in this form rather vague) question to determine the 'typical' value of $\kappa(S, G)$ for a random $k$-subset of a (perhaps specifically cyclic) group of order $n$, with $k=k(n)$ a function of $n$. 
Remark 4.3. It is easy to adapt an argument of Newman [38] to give an alternative proof of Theorem 4.1. Newman makes the nice observation (Lemma 1 in [38]) that if $A_{1}, \ldots, A_{r}$ are $a$-element subsets of some groundset $G$ such that no element of $G$ appears in more than $b$ of the $A_{i}$, and $G_{p}$ is the random set formed by selecting each element of $G$ independently with probability $p$, then the probability that $G_{p}$ meets every $A_{i}$ is at most $\left(1-(1-p)^{a}\right)^{\lceil r / b\rceil}$. Taking $A_{x}=T^{-1} x$ for each $x \in G$, and applying this with $r=n$ and $a=b=t$, one obtains the stronger bound

$$
\mathbb{P}(T S=G) \leq\left(1-(1-p)^{t}\right)^{n / t} \leq \exp (-\mu / t)
$$

in place of (4.5). The reason for writing the argument as we did is that we shall need an extension, Theorem 4.7 below; our proof of Theorem 4.1 adapts easily to give this, while Newman's argument does not seem to.

Remark 4.4. Returning to the SET COVER problem mentioned in Remark 3.4, a referee has raised the interesting question of whether there is a connection between (the proof of) Theorem 4.1 and inapproximability results for SET COVER, in particular the result of Feige [15] giving a lower bound of $(1+o(1)) \log k$ for the approximation ratio modulo certain complexity theoretic assumptions. Here we have no answers.

Perhaps the main interest of Theorem 4.1 is that it shows, together with Corollary [3.2, that the overall 'worst-case' covering multiplicity $\kappa(S, G)$ for sets of size $k$ is asymptotically $(1-o(1)) \log k$. In fact, calculating slightly more carefully in the proof of Theorem 4.1, taking $n=\lfloor k \log k\rfloor$ as before and choosing $t$ so that $t k / n=\log k-7 \log \log k+O(1)$, we can find $k$-element sets with $\kappa(S, G) \geq \log k-O(\log \log k)$, giving the following result.

Theorem 4.5. Let $\sigma_{k}$ denote the supremum of $\kappa(S, G)$ over all k-element subsets $S$ of all finite groups $G$. Then

$$
\log k-O(\log \log k) \leq \sigma_{k} \leq \log k+1 .
$$

Remark 4.2 suggests that the lower bound above can be improved, leading to the following question.

Question 4.6. What is the order of the difference $\log k-\sigma_{k}$ ? In particular, is it true that $\sigma_{k}=\log k-O(1)$ ?

In the proof of Theorem 4.1, we generated $S \subseteq G$ by selecting uniformly from all elements of $G$. Perhaps surprisingly, this is not essential: the following extension shows that we can start from any subset of $G$, as long as its size is much larger than the number $k$ of elements we pick.

Theorem 4.7. Let $0<\delta<1$ be given, and suppose that $n=n(k)$ and $h=h(k)$ are such that $n \geq h, h / k \rightarrow \infty$, and $n \leq k^{1+\delta / 7}$. If $k$ is large enough then, given any group $G$ of order $n$ and any subset $H$ of $G$ with $|H|=h$, for most $k$-element subsets $S$ of $H$ we have $\kappa(S, G) \geq(1-\delta) \log k$. 
More precisely, as $k \rightarrow \infty$, this holds for a fraction $1-o(1)$ of all $k$-element subsets $S$ of $H$.

Proof. We modify the proof of Theorem 4.1 very slightly. As before, choose $t=t(k) \sim(n / k)(1-2 \delta / 3) \log k$; the assumption on $n$ ensures that $t \leq k^{\delta / 6}$ for $k$ large.

This time, set $p=k / h=o(1)$, and form $S$ by selecting elements of $H$ independently with probability $p$, so $|S|$ again has a binomial distribution with mean $k$, and $\mathbb{P}(|S| \geq k) \geq 1 / 2$.

For $y \in H$ let $I_{y}$ be the indicator function of the event that $y \notin S$, and, as before, for $x \in G$, let $U_{x}$ denote the indicator function of the event that $x$ is not covered by $T S$. This time

$$
U_{x}=\prod_{z \in T: z^{-1} x \in H} I_{z^{-1} x}
$$

so $\mathbb{E}\left(U_{x}\right)=(1-p)^{a_{x}}$, where $a_{x}=\left|H \cap T^{-1} x\right|$. Note that

$\sum_{x} a_{x}=\left|\left\{(x, z): x \in G, z \in T, z^{-1} x \in H\right\}\right|=|\{(y, z): y \in H, z \in T\}|=t h$.

With $N=\sum_{x} U_{x}$ the number of uncovered points as before, by convexity we have

$$
\begin{aligned}
\mu & =\mathbb{E}(N)=\sum_{x}(1-p)^{a_{x}} \geq n(1-p)^{\sum_{x} a_{x} / n}=n(1-p)^{t h / n} \\
& =n \exp (-(1-o(1)) p t h / n) .
\end{aligned}
$$

Since $p t h / n=t k / n$, this yields the same lower bound (4.3) that we obtained in the proof of Theorem 4.1. The estimate (4.4) is valid as is, so the rest of the proof of Theorem 4.1 carries over unmodified.

We close this section by noting a simple consequence of Theorem 4.1 .

Example 4.8. By Theorem 4.1, for every sufficiently large $k$ there exists an $n$ and a set $S \subset \mathbb{Z}_{n}$ with $|S|=k$ such that $\kappa\left(S, \mathbb{Z}_{n}\right) \geq \log k / 2$. Consider the product set $S \times S \subset \mathbb{Z}_{n} \times \mathbb{Z}_{n}$. By Corollary [3.2, if $k$ is large enough, then

$$
\kappa\left(S \times S, \mathbb{Z}_{n} \times \mathbb{Z}_{n}\right) \leq \log |S \times S|+1=2 \log k+1<(\log k / 2)^{2} \leq \kappa\left(S, \mathbb{Z}_{n}\right)^{2} .
$$

This gives another example where the first inequality in (3.11) is strict.

$$
\text { 5. } G=\mathbb{Z}
$$

In this section we consider coverings of $G=\mathbb{Z}$, which is perhaps the simplest non-compact group. In 1954, Lorentz [35] and Erdös [12] considered coverings of $\mathbb{Z}$ by translates of an infinite subset $S$; here we shall only consider coverings by finite, non-empty subsets $S \subset \mathbb{Z}$, first studied by Newman 38]. (Actually, all three papers concern coverings of the natural numbers rather than $\mathbb{Z}$; this makes no difference.) In this case, trivially, 
$\tau(S, \mathbb{Z})=\infty$, so to make sense of the notion of covering efficiency we need to modify the definitions somewhat.

Let $\tau(S, n)$ be the smallest number of translates of $S$ that cover the set $[n]:=\{1, \ldots, n\}$, i.e.,

$$
\tau(S, n):=\min \{|T|: T+S \supseteq[n]\} .
$$

Obviously, the number of translates required to cover any other interval of integers of length $n$ is the same. It follows immediately that $\tau(S, n)$ is subadditive:

$$
\tau(S, m+n) \leq \tau(S, n)+\tau(S, m)
$$

for all $m, n \geq 1$. By a well-known result, this implies the existence of the limit

$$
\tau(S):=\lim _{n \rightarrow \infty} \tau(S, n) / n
$$

and the equality

$$
\tau(S)=\inf _{n \geq 1} \tau(S, n) / n .
$$

We call $\tau(S)$ the covering density of $S$. Newman [38] used the term 'codensity' for the minimum density $c(S)$ of a set $T \subseteq \mathbb{Z}$ with a defined density such that $T+S=\mathbb{Z}$. It is easy to see that $\tau(S)$ and $c(S)$ are equal; our more concrete definitions will be useful later. (Schmidt and Tuller [48; 49] call $c(S)$ the 'minimal covering frequency', and use 'minimal covering density' for our $\kappa(S)$ below.)

In analogy with (2.1), we define the finite covering multiplicity

$$
\kappa(S, n):=\frac{\tau(S, n)|S|}{n},
$$

which, for large $n$, is essentially the average number of translates covering each point in an optimal covering of $[n]$, the (asymptotic) covering multiplicity

$$
\kappa(S):=\lim _{n \rightarrow \infty} \kappa(S, n)=\tau(S)|S|,
$$

and the efficiency of $S$

$$
\varepsilon(S):=1 / \kappa(S) .
$$

We sometimes use the notation $\tau(S, \mathbb{Z})$ etc. for emphasis and clarity.

For a finite, non-empty $S \subset \mathbb{Z}$ we trivially have

$$
\frac{n}{|S|} \leq \tau(S, n) \leq n
$$

and thus

$$
\begin{aligned}
1 /|S| & \leq \tau(S) \leq 1, \\
1 & \leq \kappa(S) \leq|S|, \\
|S|^{-1} & \leq \varepsilon(S) \leq 1 .
\end{aligned}
$$


We shall improve these bounds in Theorem 6.4 below. Again, there are many examples of efficient sets $S$ with $\kappa(S)=\varepsilon(S)=1$, as in Example6.3. but we are mainly interested in the others.

Let $\alpha_{k}$ be the minimal efficiency over $k$-element subsets of $\mathbb{Z}$, i.e.,

$$
\alpha_{k}:=\inf \{\varepsilon(S, \mathbb{Z}):|S|=k\} .
$$

Note that $k^{-1} \leq \alpha_{k} \leq 1$, where the lower bound will be improved later. It is an interesting problem to find $\alpha_{k}$ exactly for small $k$. Trivially, $\alpha_{1}=$ 1. It is easy to see that $\alpha_{2}=1$ too. Indeed, suppose that $S \subset \mathbb{Z}$ with $|S|=2$. Translating if necessary, we may assume that $S=\{0, a\}$ with $a \geq 1$. Then $\{S+i: 0 \leq i<a\}$ is a partition of $\{0, \ldots, 2 a-1\}$, and thus $\mathcal{T}:=\{S+i+2 a j: 0 \leq i<a, j \in \mathbb{Z}\}$ is a partition of $\mathbb{Z}$. It follows that $\varepsilon(S)=1$. Hence, $\alpha_{2}=1$.

Newman [38] showed that $\alpha_{3}=5 / 6$, and (according to [54]) conjectured that $\alpha_{4}=3 / 4$; Weinstein [54] showed that $\alpha_{4} \leq 3 / 4$, and used a computer to prove a lower bound of $0.7354 \ldots$ Checking all subsets $S$ of a small interval suggests the values $\alpha_{4}=3 / 4, \alpha_{5}=11 / 5$ and $\alpha_{6}=2 / 3$; see Remark 5.6.

We may talk about the efficiency etc. of a particular covering $\mathcal{T}=\left\{t_{i}+S\right\}$ of $\mathbb{Z}$ or $\mathbb{Z}^{+}$too. We define

$$
\tau(\mathcal{T}, n):=\left|\left\{i: 0 \leq t_{i}<n\right\}\right|,
$$

and, provided the limit exists, the covering density

$$
\tau(\mathcal{T}):=\lim _{n \rightarrow \infty} \tau(\mathcal{T}, n) / n,
$$

the covering multiplicity

$$
\kappa(\mathcal{T}):=\tau(\mathcal{T})|S|
$$

and the covering efficiency

$$
\varepsilon(\mathcal{T}):=\frac{1}{\kappa(\mathcal{T})}=\frac{1}{\tau(\mathcal{T})|S|}
$$

Actually, we shall only use these notions when $\mathcal{T}$ is periodic; in this case the limit exists and, if $\mathcal{T}$ has period $\ell$, then

$$
\tau(\mathcal{T})=\tau(\mathcal{T}, \ell) / \ell
$$

From the definitions above, it is not immediately clear that there is an infinite covering $\mathcal{T}$ with $\kappa(\mathcal{T})=\kappa(S)$. However, the reader will not fall off her chair on learning that this is indeed the case. In fact, unsurprisingly, there is always an optimal periodic covering; see, for example, Schmidt and Tuller [48]. We shall give a different proof that gives a better bound on the period, and, perhaps more importantly, leads to a practical algorithm for calculating $\kappa(S)$.

Suppose without loss of generality that $S \subseteq[0, s], s \geq 1$, with $0 \in S$. Suppose that for some $N$, which we think of as large, $T \subset \mathbb{Z}$ is such that $T+S$ covers $[N]$, with $|T|$ minimal, i.e., $|T|=\tau(S, N)$. Note that $\min T \geq-s$. 
For $0 \leq m \leq N$, let $T_{m}=\{t \in T: t \leq m\}$. Then $T_{m}+S$ covers $[m]$, and $\max \left(T_{m}+S\right) \leq m+s$. Let $A_{m}=\left(T_{m}-m+s\right) \cap[s]$, so $A_{m}$ records which of the last $s$ possible elements of $T_{m}$ are in fact present. Writing $\sigma$ for the 0/1-sequence corresponding to $T$, note that each $A_{m}$ corresponds to a block of $s$ consecutive terms of $\sigma$.

Passing from $m$ to $m+1 \leq N$, there are two possibilities. In the first case $m+1 \notin T$, so $T_{m+1}=T_{m}$ and $A_{m+1}=\left(A_{m}-1\right) \backslash\{0\}$. In this case $S+T_{m}=S+T_{m+1}$ must cover $m+1$, so we must have $s+1 \in A_{m}+S$. In the second case, $m+1 \in T$, so $A_{m+1}=\left(A_{m}-1\right) \backslash\{0\} \cup\{s\}$.

Let $G_{S}$ be the weighted directed graph defined as follows. For the vertex set $V$ we take the power set $\mathcal{P}([s])$ of $\{1,2, \ldots, s\}$. Send a directed edge from $A$ to $B$ with weight 0 if and only if $s+1 \in A+S$ and $B=(A-1) \backslash\{0\}$, and send a directed edge from $A$ to $B$ with weight 1 if $B=(A-1) \backslash\{0\} \cup\{s\}$. Given a (directed) walk $\gamma$ in $G_{S}$, let us write $|\gamma|$ for its length (number of edges), and $w(\gamma)$ for its weight, i.e., the sum of the weights of its edges. Then $\gamma=A_{0} A_{1} \cdots A_{N}$ is a walk in $G_{S}$ with $|\gamma|=N$ and $w(\gamma)=\sum_{m=0}^{N-1}\left|T_{m+1}\right|$ $T_{m}|=| T \backslash T_{0} \mid$. Since $\min T \geq-s$, we have $w(\gamma)=|T|+O(1)$.

The de Bruijn graph associated to binary sequences of length $s \geq 1$ is the 2-in, 2-out directed graph whose vertices are binary sequences of length $s$, with an edge from $A$ to $B$ if the last $s-1$ terms of $s$ agree with the first $s-1$ terms of $B$, i.e., if $A$ and $B$ can appear as subsequences of $s$ consecutive terms in a single (infinite) binary sequence $\sigma$, with $B$ starting one place later than $A$. This graph was introduced independently by de Bruijn [11] and Good [20] in 1946. It is well known and easy to check that directed $n$-edge walks in the de Bruijn graph correspond bijectively to binary sequences of length $s+n$.

Identifying subsets of $[s]$ with binary sequences of length $s$ in the usual way, the unweighted directed graph underlying $G_{S}$ is a subgraph of the de Bruijn graph. It is not hard to check that, given a walk $\gamma$ in $G_{S}$ with $|\gamma|=N$, one can construct a set $T$ of size at most $w(\gamma)+s$ so that $T+S$ covers $[N]$. We omit the details since we only need the cycle case, which we prove below. As usual, by a cycle in a directed graph $G$ we mean a directed walk $v_{0} v_{1} \cdots v_{n-1} v_{0}$ in $G$ in which the vertices $v_{0}, \ldots, v_{n-1}$ are distinct.

Theorem 5.1. Let $G_{S}$ be the directed graph defined above, and let $\alpha=\alpha(S)$ be the minimum of $w(\gamma) /|\gamma|$ over all cycles $\gamma$ in $G_{S}$. Then $\tau(S)=\alpha$, and there is a periodic covering $\mathcal{T}$ with $\tau(\mathcal{T})=\tau(S)$. In particular, $\tau(S)$ is rational for every finite, non-empty $S \subset \mathbb{Z}$.

Proof. Suppose first that $T+S \supseteq[N]$, with $|T|$ minimal. We have shown that there is a walk $\gamma$ with $|\gamma|=N$ and $w(\gamma)=\left|T \backslash T_{0}\right| \leq|T|$. Now $\gamma$ may be written as a union of cycles together with a union of vertex disjoint paths. It follows that $w(\gamma) \geq \alpha(N-|V|) \geq \alpha N-2^{s}$. Hence $\tau(S, N) \geq \alpha N-2^{s}$. Letting $N \rightarrow \infty$, we see that $\tau(S) \geq \alpha$.

Conversely, let $\gamma=A_{0} A_{1} \ldots A_{\ell}$ be a cycle in $G_{S}$ with length $\ell$ and weight $w(\gamma)=\alpha \ell$. Set $A_{k+\ell m}=A_{k}$ for all $0 \leq k<\ell$ and $m \in \mathbb{Z}$, and let $T$ be 
the set of $k \in \mathbb{Z}$ such that $A_{k} A_{k+1}$ has weight 1 . Then $\left(A_{k}\right)$ is a 2-way infinite walk in $G_{S}$, and hence in the de Bruijn graph, and each $A_{k}$ codes the intersection of $T$ with the corresponding interval of length $s$. For each $k$, the fact that $A_{k} A_{k+1} \in E\left(G_{S}\right)$ ensures that $T+S$ covers $k+1$. Clearly, $\mathcal{T}=\{t+S: t \in T\}$ is periodic with density $\alpha$, so we have $\tau(S) \leq \tau(\mathcal{T})=\alpha$, completing the proof.

Let $\ell(S)$ denote the minimum period of an optimal periodic covering of $\mathbb{Z}$ by translates of $S$, and let $\ell(s)$ denote the maximum of $\ell(S)$ over all sets $S \subseteq[0, s]$, i.e., over all subsets of $\mathbb{Z}$ with diameter at most $s$. Schmidt and Tuller [48] show that $\ell(s) \leq s\left(2^{s}+1\right)$, and state that an improvement of this bound would be of interest. The proof of Theorem 5.1 shows that $\ell(S) \leq\left|G_{S}\right|=2^{s}$; in fact, we can show a little more.

Theorem 5.2. Suppose that $S \subseteq[0, s]$. Then

$$
\ell(S) \leq \min \left\{2^{s}, 2 \sum_{t \leq 2 s \tau(S)}\left(\begin{array}{l}
s \\
t
\end{array}\right)\right\} .
$$

Furthermore, there is a function $f(k)$ tending to 0 as $k \rightarrow \infty$ such that $\ell(S) \leq 2^{s f(k)}$ for all $S \subseteq[0, s]$ with $|S|=k$.

Proof. Let $\mathcal{T}$ be the periodic covering constructed in the proof of Theorem 5.1, corresponding to the cycle $\gamma$ in $G_{S}$. Writing $w(v)$ for the weight of a vertex $v$, i.e., the fraction of 1 s present in the corresponding $0 / 1$-sequence of length $s$, the average of $w(v)$ over the vertices in $\gamma$ is $w(\gamma) /|\gamma|=\tau(\mathcal{T})=$ $\tau(S)$. Hence, at least half the vertices have $w(v) \leq 2 \tau(S)$. Thus the length $\ell(S)$ of $\gamma$ is at most twice $\sum_{t<2 s \tau(S)}\left(\begin{array}{l}s \\ t\end{array}\right)$, the number of vertices with weight at most $2 \tau(S)$. Together with the trivial bound $\ell(S) \leq\left|G_{S}\right|=2^{s}$, this proves the first statement.

Newman [38] showed that if $|S|=k$, then $\tau(S) \leq(\log k+1) / k=o(1)$; see also Corollary 3.2 and Theorem 6.4 below. Using this, the second statement of the theorem follows from the first, noting that $\sum_{t \leq 2 s(\log k+1) / k}\left(\begin{array}{l}s \\ t\end{array}\right) \leq 2^{s f(k)}$ for some $f(k)$ that tends to 0 as $k \rightarrow \infty$.

It seems likely that the bounds in Theorem 5.2 are far from the truth. For tilings of $\mathbb{Z}$, i.e., for sets $S$ with $\kappa(S)=1$, much better bounds are known. Indeed, Biró [8] showed that $\ell(S) \leq \exp \left(s^{1 / 3+o(1)}\right)$ in this case. This suggests the following question, where we expect the answer to the second part to be positive.

Question 5.3. What is the approximate growth rate of the function $\ell(s)=$ $\max \{\ell(S): S \subseteq[0, s]\}$ ? In particular, is $\ell(s)=2^{o(s)}$ ?

Remark 5.4. Using Theorem 5.1 it is easy to give an algorithm for calculating $\tau(S)$. Of course, the upper bound $2^{s}$ on $\ell(S)$ gives a trivial algorithm, of complexity roughly $2^{2^{s}}$; considering the graph $G_{S}$ allows one to reduce this greatly. Indeed, given a vertex $v_{0}$ of $G_{S}$ and a positive integer $T$, using 
breadth-first search one can easily find inductively for all $t \leq T$ and for all $v$ the minimum weight of a walk from $v_{0}$ to $v$ of length $t$ (if there is one). Since a cycle is a walk of length at most $\left|G_{S}\right|$, considering each starting vertex separately one can thus determine the minimum average weight $\alpha$ of a cycle in time at most $O\left(\left|G_{S}\right|^{3}\right)=O\left(2^{3 s}\right)$. This is practical for moderate $s$, say $s \leq 10$. In fact, one can do a little better by considering a different graph $G_{S}^{\prime}$ : the definition is similar to that of $G_{S}$, except that a vertex now encodes $B_{m}=\left(T_{m}+S-m\right) \cap[s]$, corresponding to the subset of $[m+1, m+s]$ covered by $T_{m}+S$, rather than $A_{m}=\left(T_{m}-m+s\right) \cap[s]$. It is easy to see that $A_{m}$ determines $B_{m}$, but many possible $A_{m}$ may lead to the same $B_{m}$, so $G_{S}^{\prime}$ has fewer vertices than $G_{S}$.

The problem of finding a cycle with minimum average edge weight in a given weighted directed graph $G$ has received considerable attention: it is usually referred to as the MCMP or 'Minimum Cycle Mean Problem'. Suppose that $G$ has $n$ vertices and $m$ edges. Karp [31] gave a very elegant and easy to program algorithm with running time $O(\mathrm{~nm})$, which in the present context is $O\left(\left|G_{S}\right|^{2}\right)$. (This algorithm is very unintuitive, and the proof that it is correct requires a little work.) More complicated but more efficient algorithms have since been found; perhaps the most efficient is that of Orlin and Ahuja [40], with running time $O\left(n^{1 / 2} m \log n\right)$ when, as here, the edge weights are integers that are not too large. Having found the minimum average edge weight, one can then find the shortest cycle with this average weight by using Johnson's algorithm [29].

\begin{tabular}{|c|c|c|c|c|c|}
\hline$s$ & $\ell(s)$ & $S$ & $s$ & $\ell(s)$ & $S$ \\
\hline 0 & 1 & $\{0\}$ & 11 & 53 & $\{0,1,7,9,11\}$ \\
\hline 1 & 2 & $\{0,1\}$ & 12 & 66 & $\{0,1,8,12\}$ \\
\hline 2 & 4 & $\{0,2\}$ & 13 & 109 & $\{0,1,3,7,12,13\}$ \\
\hline 3 & 5 & $\{0,1,3\}$ & 14 & 129 & $\{0,1,12,14\}$ \\
\hline 4 & 8 & $\{0,4\}$ & 15 & 147 & $\{0,1,8,12,15\}$ \\
\hline 5 & 8 & & 16 & 147 & \\
\hline 6 & 13 & $\{0,1,4,6\}$ & 17 & 170 & $\{0,3,7,17\}$ \\
\hline 7 & 13 & & 18 & 192 & $\{0,2,3,7,18\}$ \\
\hline 8 & 27 & $\{0,1,3,8\}$ & 19 & 250 & $\{0,1,3,7,11,17,19\}$ \\
\hline 9 & 27 & & 20 & 286 & $\{0,1,4,10,12,19,20\}$ \\
\hline 10 & 45 & $\{0,2,7,10\}$ & 21 & 317 & $\{0,1,2,7,12,20,21\}$ \\
\hline
\end{tabular}

TABLE 1. The maximum over $S \subseteq[0, s] \subset \mathbb{Z}$ of the minimum period of an optimum covering by translates of $S$, with examples of sets $S$ achieving the maximum. When not listed, $S$ is as in the row above.

Using, for example, Karp's algorithm and then Johnson's algorithm, one can fairly easily search over all $S$ with diameter $s \leq 21$, say, to obtain the information in Table 1. Here the third column gives an example of a set $S$ 
attaining the maximum. To us, sadly, this table does not strongly suggest any particular asymptotic form of $\ell(s)$ !

Remark 5.5. Writing $\ell(s, k)$ for $\max \{\ell(S): S \subseteq[0, s],|S|=k\}$, in the light of Theorem 5.2, to show that $\ell(s)=2^{o(s)}$ it suffices to show that $\ell(s, k)=2^{o(s)}$ for each fixed $k$. However, while the numerical data in Table 2 strongly suggests this holds when $k=3$, even for $k=4$ the data is much less clear! For $k=3$, Schmidt and Tuller [48] have conjectured a simple formula for $\tau(\{0, a, b\})$ with $0<a<b$ and $a$ and $b$ coprime. If this formula is correct, then $\ell(s, 3) \leq 2 s$ for all $s$.

\begin{tabular}{|c||c|c|c|c|c|c|c|c|c|c|c|}
\hline$s$ & 3 & 4 & 5 & 6 & 7 & 8 & 9 & 10 & 11 & 12 & 13 \\
\hline$\ell(s, 3)$ & 5 & 5 & 8 & 11 & 11 & 11 & 17 & 17 & 20 & 23 & 23 \\
\hline$\ell(s, 4)$ & & 7 & 8 & 13 & 13 & 27 & $(26)$ & 45 & $(35)$ & 66 & $(58)$ \\
\hline
\end{tabular}

\begin{tabular}{|c||c|c|c|c|c|c|c|c|c|}
\hline$s$ & 14 & 15 & 16 & 17 & 18 & 19 & 20 & 21 & 22 \\
\hline$\ell(s, 3)$ & 23 & 29 & 29 & 32 & 35 & 35 & $(32)$ & 41 & 41 \\
\hline$\ell(s, 4)$ & 129 & $(91)$ & 122 & 170 & $(95)$ & $(100)$ & 183 & $(143)$ & 185 \\
\hline
\end{tabular}

TABLE 2. Longest repeating periods for optimal periodic coverings of sets with size $k=3$ and $k=4$ contained in $[0, s]$, found using Karp's algorithm. Values in brackets are the longest for such $S$ with $0, s \in S$; in these cases there is a subset $S \subseteq[0, s-1]$ with longer period.

Remark 5.6. Returning to $\alpha_{k}$, the infimum of $\varepsilon(S, \mathbb{Z})$ over $S$ with $k$ elements, we do not have an algorithm to calculate this. The problem is that we have no bound on the diameter $\operatorname{diam}(S)=\max S-\min S$ of a set achieving (or approaching) the infimum. Again, searching small examples strongly suggests that this maximum diameter does not grow too fast with $k$; see Table 3 .

\begin{tabular}{|c|c|l|}
\hline$k$ & $\alpha_{k}$ & $S$ \\
\hline 2 & 1 & $\{0,1\}$ \\
3 & $5 / 6$ & $\{0,1,3\}$ \\
4 & $3 / 4 ?$ & $\{0,1,2,4\}$ \\
5 & $11 / 15 ?$ & $\{0,1,3,4,8\}$ \\
6 & $2 / 3 ?$ & $\{0,1,2,4,6,9\}$ \\
\hline
\end{tabular}

TABLE 3. Known and conjectured values of $\alpha_{k}$ for small $k$, with examples of corresponding sets $S$; the values with question marks are upper bounds, given by the minimum over all $k$-element subsets $S$ of $[0,22]$. 
Question 5.7. Are the values in Table 3 correct? In particular, is $\alpha_{4}$ equal to 3/4, as conjectured by Newman (see Weinstein [54])?

Although it is not our focus here, let us remark that the question of which subsets $S$ tile $\mathbb{Z}$ has received much attention. Formally, $S$ tiles $\mathbb{Z}$ if there exists a set $T$ such that every integer has a unique expression as $t+s, t \in T$, $s \in S$. In the light of Theorem 5.1, this is equivalent to $\kappa(S)=1$. When $|S|$ is a prime power, Newman [39] gave a simple necessary and sufficient condition for $S$ to tile $\mathbb{Z}$; Coven and Meyerowitz [10] answered the question when $|S|$ has two prime factors. In the general case, there are only partial results; see, for example, Konyagin and Laba [32].

\section{LINEAR VS. CYCLIC}

If $S \subset \mathbb{Z}$ is finite and $n$ is sufficiently large, say $n>\operatorname{diam}(S)$, then we can regard $S$ as a subset of $\mathbb{Z}_{n}=\mathbb{Z} / n \mathbb{Z}$. Clearly, if $T \subseteq \mathbb{Z}$ is a set such that $T+S \supseteq[n]$, and $\widehat{T}$ is the image of $T$ in $\mathbb{Z}_{n}$, then $\widehat{T}+\bar{S}=\mathbb{Z}_{n}$ and $|\widehat{T}| \leq|T|$. Thus

$$
\tau\left(S, \mathbb{Z}_{n}\right) \leq \tau(S, n)
$$

Equality does not necessarily hold, see Example 6.2 below, but the difference is negligible for $S$ fixed and $n$ large, as shown by the following lemma.

Lemma 6.1. Let $S \subset \mathbb{Z}$ be a finite set and $n>\operatorname{diam}(S)$. Then

$$
\tau(S, \mathbb{Z}) \leq \frac{\tau\left(S, \mathbb{Z}_{n}\right)}{n} \leq \frac{\tau(S, n)}{n}
$$

and

$$
\kappa(S, \mathbb{Z}) \leq \kappa\left(S, \mathbb{Z}_{n}\right) \leq \kappa(S, n) .
$$

More precisely, for any $m \geq 1$, with $d=\operatorname{diam}(S)$,

$$
\tau(S, m n-d) \leq m \tau\left(S, \mathbb{Z}_{n}\right) .
$$

Furthermore, for any finite $S \subset \mathbb{Z}$,

$$
\begin{aligned}
\lim _{n \rightarrow \infty} \frac{\tau\left(S, \mathbb{Z}_{n}\right)}{n} & =\tau(S, \mathbb{Z})=\inf _{n \geq 1} \frac{\tau\left(S, \mathbb{Z}_{n}\right)}{n}, \\
\lim _{n \rightarrow \infty} \kappa\left(S, \mathbb{Z}_{n}\right) & =\kappa(S, \mathbb{Z})=\inf _{n \geq 1} \kappa\left(S, \mathbb{Z}_{n}\right), \\
\lim _{n \rightarrow \infty} \varepsilon\left(S, \mathbb{Z}_{n}\right)=\varepsilon(S, \mathbb{Z}) & =\sup _{n \geq 1} \varepsilon\left(S, \mathbb{Z}_{n}\right) .
\end{aligned}
$$

Proof. Let $S$ be a finite subset of $\mathbb{Z}$ with diameter $d$. By translating $S$, which does not change any of the numbers studied here, we may and shall assume that $S \subseteq[0, d]$. For $n>d$, regarding $S$ as a subset of $\mathbb{Z}_{n}$ as above, let $\{\hat{t}+S: \hat{t} \in \widehat{T}\}$ be an optimal covering of $\mathbb{Z}_{n}$ by translates of $S$. Choosing a representative $t \in[0, n-1] \subset \mathbb{Z}$ for each $\hat{t} \in \widehat{T}$, we obtain a set $T \subseteq[0, n-$ 1] $\subset \mathbb{Z}$ with $|T|=|\widehat{T}|=\tau\left(S, \mathbb{Z}_{n}\right)$ such that $T+S$ contains a representative of every residue class modulo $n$. Since $S \subseteq[0, d]$, we have $T+S \subseteq[0, n+d-1]$. Since $n>d$, this interval contains at most two representatives of each residue 
class modulo $n$, and it follows that $T+S \supseteq[d, n-1]$, and that if $x \in[0, d-1]$, then $x \in T+S$ or $x+n \in T+S$ (or both). For $m \geq 1$, let $T_{m}:=T+\{j n$ : $0 \leq j<m\}$; it follows then that $T_{m}+S \supseteq[d, m n-1]$. Consequently,

$$
\tau(S, m n-d) \leq\left|T_{m}\right|=m|T|=m \tau\left(S, \mathbb{Z}_{n}\right),
$$

which proves (6.4).

Dividing (6.4) by $m n-d$ and letting $m \rightarrow \infty$ we obtain the first inequality in (6.2); the second follows by (6.1). Next, (6.2) implies (6.5) by (5.2) and (5.3). The remaining statements then follow from the definitions: multiplying (6.2) and (6.5) by $|S|$ we obtain (6.3) and (6.6) by (2.1), (5.4), (5.5). Finally, (6.7) follows by taking reciprocals.

Example 6.2. If $S=\{0,1,5\}$, then $\tau\left(S, \mathbb{Z}_{6}\right)=2$ because $S \cup(S+3)=$ $\mathbb{Z}_{6}$ (see also Example 6.3 below), but it is impossible to cover the interval $[0,5] \subset \mathbb{Z}$ by two translates of $S$ and thus $\tau(S, 6)>2$. (In fact, it is easily seen that $\tau(S, 6)=3$.)

Example 6.3. If $S \subset \mathbb{Z}$ contains exactly one element from each residue class modulo $k:=|S|$, then, for every $m \geq 1$, working in $\mathbb{Z}_{m k}$ we have $\{j k: 0 \leq$ $j<m\}+S=\mathbb{Z}_{m k}$ and thus $\tau\left(S, \mathbb{Z}_{m k}\right)=m=\left|\mathbb{Z}_{m k}\right| /|S|$ and $\kappa\left(S, \mathbb{Z}_{m k}\right)=1$. Consequently, by Lemma 6.1, $\tau(S)=1 /|S|$ and $\kappa(S)=\varepsilon(S)=1$.

Our next result slightly improves Newman's result [38] for coverings of the natural numbers.

Theorem 6.4. If $S \subseteq \mathbb{Z}$ is finite with $|S|=k \geq 1$, then

$$
\begin{aligned}
& \kappa(S) \leq H_{k} \leq \log k+1, \\
& \varepsilon(S) \geq \frac{1}{H_{k}} \geq \frac{1}{\log k+1} .
\end{aligned}
$$

In other words, $\alpha_{k}$, the minimal efficiency of a $k$-set, is at least $1 / H_{k}$.

Proof. An immediate consequence of (6.6) and (3.7).

Lemma 6.1 shows that $\mathbb{Z}$ is 'easier' to cover efficiently than $\mathbb{Z}_{n}$. The next lemma shows that the difference is not that large.

Lemma 6.5. Let $S \subset \mathbb{Z}$ have diameter $d<\infty$. Then for any $n>d$ we have

$$
\kappa\left(S, \mathbb{Z}_{n}\right) \leq \frac{n+d}{n} \kappa(S, \mathbb{Z})<2 \kappa(S, \mathbb{Z}) .
$$

Proof. Without loss of generality, suppose that $\min S=0$, so $\max S=d$. Let $\mathcal{T}=\{t+S: t \in T\}$ be a covering with $\kappa(\mathcal{T})=\kappa(s, \mathbb{Z})$. By Theorem 5.1 such a $\mathcal{T}$ exists, and we may assume that $\mathcal{T}$ is periodic. (We do not need this here, and could just as well work with an optimal covering of $[\mathrm{m}]$ for $m$ large.)

For $a \in \mathbb{Z}$ let $I_{a}=[a, a+n-1]$, an interval of length $n$. Then $t+S$ meets $I_{a}$ if and only if $t \in J_{a}=[a-d, a+n-1]$, an interval of length $n+d$. Hence, $T_{a}+S \supset I_{a}$, where $T_{a}=T \cap J_{a}$. Since $\mathcal{T}$ (which is periodic) 
has density $\tau(S, \mathbb{Z})=\kappa(S, \mathbb{Z}) /|S|$, a simple averaging argument shows that there is some $a$ with $\left|T_{a}\right| \leq\left|J_{a}\right| \kappa(S, \mathbb{Z}) /|S|=(n+d) \kappa(S, \mathbb{Z}) /|S|$. In $\mathbb{Z}_{n}$ we have $\widehat{T}_{a}+S \supset \widehat{I}_{a}=\mathbb{Z}_{n}$, so $\tau\left(S, \mathbb{Z}_{n}\right) \leq(n+d) \kappa(S, \mathbb{Z}) /|S|$ and the result follows.

Combined with Theorem 4.7. Lemma 6.5 gives a new proof of Newman's result [38] concerning the asymptotic value of $\alpha_{k}$, the minimal efficiency of a $k$-set.

Theorem 6.6. We have $\alpha_{k} \sim 1 / \log k$ as $k \rightarrow \infty$.

Proof. In the light of Theorem 6.4, we need only prove that $\alpha_{k} \leq(1+$ $o(1)) / \log k$. Given $\delta>0$, set $h=h(k)=\lceil k \log k\rceil$ and $n=n(k)=$ $\left\lceil k(\log k)^{2}\right\rceil$, say. Then the assumptions of Theorem 4.7 are satisfied. Applying this result to $[h] \subset \mathbb{Z}_{n}$, it follows that for $k$ large enough, there is some $S \subset[h]$ with $|S|=k$ such that $\kappa\left(S, \mathbb{Z}_{n}\right) \geq(1-\delta) \log k$. Since $\operatorname{diam}(S) \leq h$ and $h / n \rightarrow 0$, from Lemma 6.5 it follows that

$$
\kappa(S, \mathbb{Z}) \geq \kappa\left(S, \mathbb{Z}_{n}\right) \frac{n}{n+h} \geq(1-\delta-o(1)) \log k .
$$

Since $\delta>0$ is arbitrary, it follows that $\sup \{\kappa(S, \mathbb{Z}):|S|=k\} \geq(1-$ $o(1)) \log k$ as $k \rightarrow \infty$. In other words, $\alpha_{k} \leq(1+o(1)) / \log k$.

\section{SMALl SETS IN $\mathbb{Z}$ AND $\mathbb{Z}_{n}$}

In this section we shall consider small sets $S$ in either $\mathbb{Z}$ or $\mathbb{Z}_{n}$ ( $n$ large), with the aim of showing that for 'typical' sets $S$ of some fixed size $k$, the efficiency is close to 1 . Given an integer $x \in \mathbb{Z}$, we sometimes write $x$ for the corresponding element in $\mathbb{Z}_{n}$, but in this section for clarity we usually denote the latter by $\bar{x}$. Thus $\bar{x}=\bar{y} \Longleftrightarrow x \equiv y(\bmod n)$.

Schmidt [47] proved results relating the covering multiplicity of $S$ to the linear equations satisfied by its elements, showing that if the elements satisfy no non-trivial equations with small coefficients, then $S$ covers efficiently. Restated in our terminology, these results are as follows.

Theorem 7.1 (47]). For every $k \geq 2$ and $\varepsilon>0$ there exists a constant $C=C(k, \varepsilon)$ such that for every $n \geq k$, if $S=\left\{\bar{x}_{1}, \ldots, \bar{x}_{k}\right\}$ is a $k$-subset of the cyclic group $\mathbb{Z}_{n}$ with $\kappa\left(S, \mathbb{Z}_{n}\right) \geq 1+\varepsilon$, then there exist integer coefficients $a_{1}, \ldots, a_{k}$, with $\sum_{i=1}^{k} a_{i}=0$ and $0<\max _{i}\left|a_{i}\right| \leq C$, such that

$$
\sum_{i=1}^{k} a_{i} \bar{x}_{i}=\sum_{i=1}^{k-1} a_{i}\left(\bar{x}_{i}-\bar{x}_{k}\right)=0
$$

in $\mathbb{Z}_{n}$.

Theorem 7.2 ([47]). For every $k \geq 2$ and $\varepsilon>0$ there exists a constant $C=C(k, \varepsilon)$ such that if $S=\left\{x_{1}, \ldots, x_{k}\right\}$ is a $k$-subset of $\mathbb{Z}$ with $\kappa(S, \mathbb{Z}) \geq$ 
$1+\varepsilon$, then there exist integer coefficients $a_{1}, \ldots, a_{k}$ with $\sum_{i=1}^{k} a_{i}=0$ and $0<\max _{i}\left|a_{i}\right| \leq C$, such that

$$
\sum_{i=1}^{k} a_{i} x_{i}=0 .
$$

Although Schmidt proved his results above in a different order, it is easy to see that Theorem 7.1 implies Theorem 7.2 .

Proof. Let $C=C(k, \varepsilon)$ be as in Theorem 7.1. Given $S=\left\{x_{1}, \ldots, x_{k}\right\} \subset$ $\mathbb{Z}$, choose any integer $n>C \sum_{i=1}^{k}\left|x_{i}\right|$ and let $\bar{S}=\left\{\bar{x}_{1}, \ldots, \bar{x}_{k}\right\} \subseteq \mathbb{Z}_{n}$. Then $\kappa\left(\bar{S}, \mathbb{Z}_{n}\right) \geq \kappa(S, \mathbb{Z})$ by (6.3). Hence Theorem 7.1 gives $\sum_{i=1}^{k} a_{i} x_{i} \equiv 0$ $(\bmod n)$ for some $a_{i}$ with $\sum_{i=1}^{k} a_{i}=0$ and $0<\max _{i}\left|a_{i}\right| \leq C$. From the choice of $n$ it follows that $\sum_{i=1}^{k} a_{i} x_{i}=0$.

Theorems 7.1 and 7.2 easily imply that 'typical' sets cover efficiently; let us make this precise. We consider $\mathbb{Z}_{n}$ first. As usual, $\stackrel{\mathrm{p}}{\longrightarrow}$ denotes convergence in probability.

Corollary 7.3. Let $S_{n, k}$ denote a k-element subset of $\mathbb{Z}_{n}$ chosen uniformly at random. For every fixed $k$ we have $\varepsilon\left(S_{n, k}, \mathbb{Z}_{n}\right) \stackrel{\mathrm{p}}{\longrightarrow} 1$ as $n \rightarrow \infty$.

Proof. With $k$ fixed, let $\tilde{S}=\tilde{S}_{n, k}:=\left\{\bar{x}_{1}, \ldots, \bar{x}_{k}\right\}$, where $\bar{x}_{1}, \ldots, \bar{x}_{k}$ are independent uniformly random elements of $\mathbb{Z}_{n}$. Then $S_{n, k} \stackrel{\mathrm{d}}{=}(\tilde{S}|| \tilde{S} \mid=k)$, and since $\mathbb{P}(|\tilde{S}| \neq k) \leq k^{2} / n=o(1)$, it suffices to consider $\tilde{S}$.

Let $\varepsilon>0$ and apply Theorem 7.1. For every fixed $\left(a_{1}, \ldots, a_{k}\right) \neq(0, \ldots, 0)$, the sum $\sum_{i} a_{i} \bar{x}_{i}$ is uniformly distributed on $\mathbb{Z}_{n}$, and thus $\mathbb{P}\left(\sum_{i} a_{i} \bar{x}_{i}=0\right)=$ $1 / n$. Hence the probability that one of the at most $(2 C+1)^{k}$ possible conditions (7.1) holds is at most $(2 C+1)^{k} / n=o(1)$, and thus Theorem 7.1 yields $\mathbb{P}\left(\kappa\left(\tilde{S}, \mathbb{Z}_{n}\right) \geq 1+\varepsilon\right) \rightarrow 0$ as $n \rightarrow \infty$. Thus $\kappa\left(S, \mathbb{Z}_{n}\right) \stackrel{\mathrm{p}}{\longrightarrow} 1$. Equivalently, $\varepsilon\left(S, \mathbb{Z}_{n}\right) \stackrel{\mathrm{p}}{\longrightarrow} 1$.

We have seen that, for fixed $k$ and large $n$, most $k$-subsets cover $\mathbb{Z}_{n}$ with close to optimal efficiency. In contrast, Theorem 4.1 and Remark 4.2 show that if $n$ is only slightly larger than $k$, then most $k$-subsets of $\mathbb{Z}_{n}$ have almost the worst possible efficiency.

Question 7.4. Where is the threshold? More precisely, for which sequences $n=n(k) \geq k$ is it true that $\varepsilon\left(S_{n, k}, \mathbb{Z}_{n}\right) \stackrel{\mathrm{p}}{\longrightarrow} 1$ as $k \rightarrow \infty$ ? For which sequences is $\varepsilon\left(S_{n, k}, \mathbb{Z}_{n}\right) \sim 1 / \log k$ ?

Our next corollary shows that 'typical' $k$-element subsets of $\mathbb{Z}$ cover efficiently. To make sense of this, we choose our subsets from a large interval $[n]=\{1,2, \ldots, n\}$, and take limits as $n \rightarrow \infty$.

Corollary 7.5. Let $S_{n, k}^{\prime}$ denote a k-element subset of $[n]$ chosen uniformly at random. For every fixed $k$ we have $\varepsilon\left(S_{n, k}^{\prime}, \mathbb{Z}\right) \stackrel{\mathrm{p}}{\longrightarrow} 1$ as $n \rightarrow \infty$. 
Proof. Using the obvious coupling between random subsets of $[n]$ and random subsets of $\mathbb{Z}_{n}$, (6.3) gives $\varepsilon\left(S_{n, k}^{\prime}, \mathbb{Z}\right) \geq \varepsilon\left(S_{n, k}, \mathbb{Z}_{n}\right)$. The result follows by Corollary 7.3 .

One can also use the results above to show that certain deterministic sequences of sets $S$ are asymptotically efficient.

Example 7.6. Fix distinct integers $a$ and $b$, and let $S=S_{n}:=\{a, b, n\}$. It is easily seen that, for any given $C$, if $n$ is large enough then the conclusion of Theorem 7.2 cannot hold for this $C$. Thus Theorem 7.2 implies that $\varepsilon\left(S_{n}, \mathbb{Z}\right) \rightarrow 1$ as $n \rightarrow \infty$. (See also [47; 52].)

In the appendix we give a new proof of Schmidt's results; it seems to us that our proof is different from that in [47]. Ours is based on the following number theoretic lemma, which may be seen as a discrete version of Kronecker's Theorem (see, for example, [24, Chapter XXIII]), which states that if $x_{1}, \ldots, x_{m}$ are real numbers that are linearly independent over $\mathbb{Q}$, then the set of multiples of $\left(x_{1}, \ldots, x_{m}\right)$ is dense in the torus $\mathbb{R}^{m} / \mathbb{Z}^{m}$. It may well be that this lemma is known, but we have not been able to find a reference, perhaps because much more attention has been paid to quantitative versions of Kronecker's Theorem than to discrete ones. As usual, we write $(x, y)$ for the greatest common divisor of two integers $x$ and $y$.

Lemma 7.7. For every $m \geq 1$ and $\delta>0$ there exists $C=C(m, \delta)$ such that for every $n$ and every $b_{1}, \ldots, b_{m} \in \mathbb{Z}$, if $\bar{x}_{1}, \ldots, \bar{x}_{m}$ are any $m$ elements of $\mathbb{Z}_{n}$, then at least one of the following holds.

(i) There exists a vanishing linear combination $\sum_{i=1}^{m} a_{i} \bar{x}_{i}=0$ in $\mathbb{Z}_{n}$ with integer coefficients $a_{1}, \ldots, a_{m}$ satisfying $0<\max _{i}\left|a_{i}\right| \leq C$.

(ii) There exist $z \in \mathbb{Z}$ with $(z, n)=1$ and $y_{1}, \ldots, y_{m} \in[0, \delta n] \cap \mathbb{Z}$ such that $z \bar{x}_{i}-\bar{b}_{i}=\bar{y}_{i}$ in $\mathbb{Z}_{n}$, i.e.,

$$
z x_{i}-b_{i} \equiv y_{i} \quad(\bmod n), \quad i=1, \ldots, m .
$$

For the proof, see the appendix.

$$
\text { 8. } G=\mathbb{R}
$$

In this section we consider coverings of the other basic non-compact group, namely $\mathbb{R}$. Of course, if the union $T+S$ of a set of translates $t+S$ of a given set $S$ covers $\mathbb{R}$, then $S$ and $T$ cannot both be discrete. There are two natural analogues of the results and problems discussed so far in this paper. In one, $S$ is discrete (typically finite), and the 'size' or 'density' of $T$ is measured using the Lebesgue measure. Such coverings are considered implicitly by, for example, Rohlin [46] and Laczkovich [33], and explicitly by Schmidt [47]. It seems closer to the spirit of the original papers of Erdös [12], Lorentz [35] and Newman [38], however, to consider cases where $T$ is discrete. Then $S$ cannot be; usually, we will take $S$ to be some 'nice' set, such as a finite union of intervals. 
We shall only consider coverings by measurable sets $S \subset \mathbb{R}$ with nonempty interior. Typically, $\tau(S, \mathbb{R})=\infty$, so we need to modify the definitions in Section 2, Since the modifications from the compact case to the case $G=\mathbb{R}$ are closely analogous to those from the finite case to the case $G=\mathbb{Z}$, we describe these changes only briefly.

Let $\lambda$ denote the Lebesgue measure. For $x>0$, let $\tau(S, x)$ be the smallest number of translates of $S$ that cover the interval $[0, x]$, i.e.,

$$
\tau(S, x):=\min \{|T|: T+S \supseteq[0, x]\} .
$$

Obviously, the number of translates required to cover any other interval of length $x$ is the same, and

$$
\tau(S, x+y) \leq \tau(S, x)+\tau(S, y)
$$

for all $x, y>0$. Hence $\tau(S, x) / x$ converges as $x \rightarrow \infty$, and the limit satisfies

$$
\tau(S):=\lim _{x \rightarrow \infty} \tau(S, x) / x=\inf _{x>0} \tau(S, x) / x .
$$

Assuming, as we do, that $S$ has non-empty interior, then this covering density $\tau(S)$ is finite.

Using (8.3) in place of (5.2) and (5.3), and working with $\lambda(S)$ in place of $|S|$, we may adapt the definitions of $\tau(S), \kappa(S)$ and $\varepsilon(S)$ as in Section 5 , We may use the notation $\tau(S, \mathbb{R})$ etc. for emphasis and clarity. Similarly, we can make sense of the efficiency of a particular covering, at least when it is periodic.

In Sections 9 and 10, we shall consider the case when $S$ is a union of $k$ closed intervals $I_{i}: S=\bigcup_{i=1}^{k} I_{i}$. The case $k=1$ is trivial; then $\tau(S, x)=$ $\lceil x / \lambda(S)\rceil$, so $\tau(S)=\lambda(S)^{-1}$ and $\kappa(S)=\varepsilon(S)=1$.

For larger $k$ we obtain a trivial bound by ignoring all but one of the intervals, $I_{j}$, say, and using the cover $\mathcal{T}:=\left\{i a_{j}+S: i \in \mathbb{Z}\right\}$ where $a_{j}:=$ $\lambda\left(I_{j}\right)$. This covering is periodic, and has density $\tau(\mathcal{T})=a_{j}^{-1}$; hence its efficiency is $\varepsilon(\mathcal{T})=a_{j} / \lambda(S)=a_{j} / \sum_{i} a_{i}$. Choosing $j$ so that $a_{j}$ is maximal, this yields

$$
\varepsilon(S) \geq \frac{\max _{i} a_{i}}{\sum_{i} a_{i}}
$$

In particular, for any set $S$ that is a union of $k$ (closed) intervals,

$$
\varepsilon(S) \geq \frac{1}{k}
$$

We define

$$
\begin{aligned}
& \beta_{k}:=\inf \left\{\varepsilon\left(\bigcup_{1}^{k} I_{i}\right): I_{i} \text { intervals in } \mathbb{R}\right\}, \\
& \gamma_{k}:=\inf \left\{\varepsilon\left(\bigcup_{1}^{k} I_{i}\right): I_{i} \text { intervals in } \mathbb{R} \text { of the same length }\right\} .
\end{aligned}
$$

Obviously, by making small magnifications of the intervals, we may restrict the intervals in (8.6) and (8.7) to be closed (or open, or half-open) without 
changing the infima. Also, we may require the intervals in (8.7) to all have unit length.

From (8.5) and trivial inequalities we have $k^{-1} \leq \beta_{k} \leq \gamma_{k} \leq 1$. The lower bound will be improved in Section 9. It is interesting to try to find $\beta_{k}$ and $\gamma_{k}$ exactly for small $k$. Trivially, $\beta_{1}=\gamma_{1}=1$; we shall prove that $\beta_{2}=\frac{2}{3}$ and $\gamma_{2}=\frac{3}{4}$ in Section 10 .

Theorem 8.1. For every $k \geq 1, \beta_{k} \leq \gamma_{k} \leq \alpha_{k} \leq 1$.

Proof. We have already observed the first inequality.

For the second, it is slightly simpler to use half-open (or open) intervals. Given $S=\left\{s_{1}, \ldots, s_{k}\right\} \subset \mathbb{Z}$, let $I_{j}=\left[s_{j}, s_{j}+1\right)$, and set $\tilde{S}:=\bigcup_{j=1}^{k} I_{j}=$ $S+[0,1)$.

Suppose that $T=\left\{t_{1}, \ldots, t_{l}\right\} \subset \mathbb{R}$ is such that $T+\tilde{S} \supseteq[0, n]$. If $m \in$ $\{1,2, \ldots, n\}$, then $m \in T+\tilde{S}$, so $m \in t_{i}+I_{j}$ for some $t_{i} \in T$ and $1 \leq j \leq k$. In other words, $m=t_{i}+s_{j}+x$ for some $t_{i} \in T, s_{j} \in S$ and $x \in[0,1)$. Hence $t_{i}+x=m-s_{j} \in \mathbb{Z}$, so $t_{i}+x=\left\lceil t_{i}\right\rceil$ and $m=\left\lceil t_{i}\right\rceil+s_{j}$. Consequently, setting $\widehat{T}:=\{\lceil t\rceil: t \in T\}$, we have $\widehat{T}+S \supseteq[n]$.

This shows that the number $\tau(S, n ; \mathbb{Z})$ of translates of $S$ needed to cover $\{1,2, \ldots, n\}$ is at most $|\widehat{T}| \leq|T|$. Minimizing over $T$, we thus have $\tau(S, n ; \mathbb{Z}) \leq$ $\tau(\tilde{S}, n ; \mathbb{R})$, where $\tau(\tilde{S}, n ; \mathbb{R})$, as in $(\underline{8.1})$, is the minimum number of translates of $\tilde{S} \subset \mathbb{R}$ needed to cover $[0, n] \subset \mathbb{R}$. From (5.3) and (8.3), it follows that

$$
\tau(S, \mathbb{Z}) \leq \tau(\tilde{S}, \mathbb{R})
$$

Since $\lambda(\tilde{S})=|S|$, the definitions (5.5) and (5.6) and their analogues for $\mathbb{R}$ then give

$$
\begin{aligned}
& \kappa(S, \mathbb{Z}) \leq \kappa(\tilde{S}, \mathbb{R}), \\
& \varepsilon(S, \mathbb{Z}) \geq \varepsilon(\tilde{S}, \mathbb{R}) \geq \gamma_{k} .
\end{aligned}
$$

Taking the infimum in (5.11) we find $\alpha_{k} \geq \gamma_{k}$.

Note that since $\alpha_{2}=1$, Corollary 10.4 in Section [10 shows that the inequalities $\beta_{k} \leq \gamma_{k} \leq \alpha_{k}$ are strict for $k=2$; we conjecture that these inequalities are strict for all larger $k$ too.

\section{IMPROVED BOUNDS FOR UNIONS OF INTERVALS IN $\mathbb{R}$}

It seems difficult to use the greedy algorithm directly on $\mathbb{R}$, since it is not obvious when a small residual uncovered set really is empty. But we can use our result for $\mathbb{Z}$, which was based on the greedy algorithm in $\mathbb{Z}_{n}$ for large $n$.

Theorem 9.1. If $S=\bigcup_{i=1}^{k} I_{i}$ is a union of $k \geq 2$ intervals in $\mathbb{R}$, then

$$
\kappa(S) \leq \log k+\log \log k+5
$$

and thus $\varepsilon(S) \geq(1-o(1)) / \log k$. 
Proof. Recall that $\lambda$ denotes the Lebesgue measure. By homogeneity, we may assume that $\lambda(S)=1$. Fix $\delta>0$ and consider the regularly spaced intervals $J_{j}:=[j \delta,(j+1) \delta]$ of length $\delta$. Let $U:=\left\{j \in \mathbb{Z}: J_{j} \subseteq S\right\}$ and set $S^{\prime}:=\bigcup_{j \in U} J_{j} \subseteq S$, so $S^{\prime}$ is the maximal subset of $S$ consisting of closed intervals all of whose endpoints are multiples of $\delta$.

Assuming, as we may, that $I_{1}, \ldots, I_{k}$ are disjoint, we have $U=\bigcup_{i=1}^{k} U_{i}$, where $U_{i}=\left\{j \in \mathbb{Z}: J_{j} \subseteq I_{i}\right\}$. Since $I_{i} \backslash \bigcup_{j \in U_{i}} J_{j}$ consists of at most two intervals (at each end of $I_{i}$ ) of lengths $<\delta$, we have $\left|U_{i}\right| \delta>\lambda\left(I_{i}\right)-2 \delta$ and thus

$$
\lambda\left(S^{\prime}\right)=|U| \delta=\sum_{i=1}^{k}\left|U_{i}\right| \delta>\sum_{i=1}^{k} \lambda\left(I_{i}\right)-2 k \delta=\lambda(S)-2 k \delta=1-2 k \delta .
$$

We will choose $\delta<1 /(2 k)$, so $S^{\prime} \neq \emptyset$.

Since the intervals $J_{j}$ have equal lengths and form a partition of $\mathbb{R}$ (except for common endpoints), it is obvious that $\tau\left(S^{\prime}, n \delta\right) \leq \tau(U, n)$. Taking limits (recalling (5.2) and (8.3) $)$, it follows that $\tau\left(S^{\prime}, \mathbb{R}\right) \leq \tau(U, \mathbb{Z}) / \delta$, so $\kappa\left(S^{\prime}, \mathbb{R}\right) \leq$ $\kappa(U, \mathbb{Z})$. Consequently, Theorem 6.4 yields

$$
\kappa\left(S^{\prime}, \mathbb{R}\right) \leq \kappa(U, \mathbb{Z}) \leq \log |U|+1=\log \frac{\lambda\left(S^{\prime}\right)}{\delta}+1 .
$$

Since $S^{\prime} \subseteq S$, we have $\tau(S, \mathbb{R}) \leq \tau\left(S^{\prime}, \mathbb{R}\right)$ and thus, using $\lambda\left(S^{\prime}\right) \leq \lambda(S)=1$ and (9.1),

$$
\kappa(S, \mathbb{R}) \leq \frac{\lambda(S)}{\lambda\left(S^{\prime}\right)} \kappa\left(S^{\prime}, \mathbb{R}\right) \leq \frac{1}{\lambda\left(S^{\prime}\right)}\left(\log \frac{\lambda\left(S^{\prime}\right)}{\delta}+1\right) \leq \frac{1}{1-2 k \delta}\left(\log \frac{1}{\delta}+1\right) .
$$

Choosing $\delta=(2 k(\log k+1))^{-1}$ (which is close to optimal), this yields

$$
\begin{aligned}
\kappa(S, \mathbb{R}) & \leq \frac{\log k+1}{\log k}(\log 2+\log k+\log (\log k+1)+1) \\
& \leq\left(1+\frac{1}{\log k}\right)\left(\log k+\log \log k+1+\log 2+\frac{1}{\log k}\right) .
\end{aligned}
$$

This bound is evidently $\log k+\log \log k+O(1)$. To be more precise, it is easily verified that the difference between the final bound in (9.5) and $(\log k+\log \log k)$ is decreasing, and a numerical calculation verifies that this difference is smaller than 5 when $k=6$, which completes the proof for $k \geq 6$. The trivial bound $\kappa(S) \leq k$ is enough when $k \leq 7$.

Together, Theorems 8.1 and 9.1 show that $(1-o(1)) / \log k \leq \beta_{k} \leq \gamma_{k} \leq$ $\alpha_{k} \leq 1$. By Theorem 6.6 , the asymptotics are the same for all three quantities: $\beta_{k} \sim \gamma_{k} \sim \alpha_{k} \sim 1 / \log k$ as $k \rightarrow \infty$.

\section{Two InTERVALS IN $\mathbb{R}$}

Let us consider the case $k=2$, when $S=I_{1} \cup I_{2}$ is a union of two closed intervals. We may assume that the intervals are disjoint, since otherwise $S$ is an interval and thus $\varepsilon(S)=1$. 
Theorem 10.1. (i) If $S=I_{1} \cup I_{2}$ where $I_{1}$ and $I_{2}$ are two closed intervals, then $\varepsilon(S)>2 / 3$.

(ii) If in addition $I_{1}$ and $I_{2}$ have the same length, then $\varepsilon(S)>3 / 4$.

Proof. We shall prove the inequalities by giving explicit constructions of coverings with these efficiencies. As we do not have a single covering algorithm, we shall consider different constructions for different cases.

Let $a:=\lambda\left(I_{1}\right)$ and $b:=\lambda\left(I_{2}\right)$ be the lengths of the intervals, and let $c>0$ be the gap between them. By symmetry we may assume that $a \geq b>0$ and that $I_{1}$ lies to the left of $I_{2}$; we also assume that the left endpoint of $I_{1}$ is 0 . Thus, $I_{1}=[0, a]$ and $I_{2}=[a+c, a+c+b]$.

Method $I$. Ignore $I_{2}$ and use only $I_{1}$ to cover by $\mathcal{T}=\{S+n a: n \in \mathbb{Z}\}$. Clearly, $\tau(\mathcal{T})=\tau\left(I_{1}\right)=a^{-1}$ and thus $\kappa(S) \leq \kappa(\mathcal{T})=(a+b) / a$ and, as in (8.4),

$$
\varepsilon(S) \geq \varepsilon(\mathcal{T})=\frac{1}{\tau(\mathcal{T}) \lambda(S)}=\frac{a}{a+b} .
$$

Method II. Assume $c \leq b$. Then $S \cup(S+b)=[0, a+c+b+b]$ is an interval of length $a+2 b+c$. We may thus cover $\mathbb{R}$ by $\mathcal{T}:=\{S+i b+j(a+2 b+c)$ : $i=0,1, j \in \mathbb{Z}\}$. This covering is periodic, with period $a+2 b+c$, and 2 translates of $S$ begin in each period. Hence $\tau(\mathcal{T})=2 /(a+2 b+c)$, and the efficiency is

$$
\varepsilon(S) \geq \varepsilon(\mathcal{T})=\frac{1}{\tau(\mathcal{T}) \lambda(S)}=\frac{a+2 b+c}{2(a+b)}, \quad c \leq b .
$$

Method III. Consider first the translates $\{S+i(a+b+c): i \in \mathbb{Z}\}$. Since the right endpoint of $S$ equals the left endpoint of $S+(a+b+c)$, these translates match up perfectly, and the union $S^{\prime}:=\bigcup_{i \in \mathbb{Z}}(S+i(a+b+c))$ consists of an infinite sequence of intervals of length $a+b$ with gaps of length $c$ in between.

Let $y:=c /(a+b)$ and let $m:=\lceil y\rceil$. Then $\bigcup_{j=0}^{m}\left(S^{\prime}+j(a+b)\right)=\mathbb{R}$, and thus $\mathcal{T}:=\{S+i(a+b+c)+j(a+b): i \in \mathbb{Z}, j=0, \ldots, m\}$ is a covering of $\mathbb{R}$. This covering is periodic, with period $a+b+c$, and $m+1$ translates of $S$ begin in each period. Hence $\tau(\mathcal{T})=(m+1) /(a+b+c)$ and the efficiency is

$$
\varepsilon(S) \geq \varepsilon(\mathcal{T})=\frac{1}{\tau(\mathcal{T}) \lambda(S)}=\frac{a+b+c}{(m+1)(a+b)}=\frac{y+1}{m+1}=\frac{y+1}{\lceil y+1\rceil} .
$$

To complete the proof of the first part, we shall show for any positive $a, b, c$ with $a \geq b$, at least one of these methods yields $\varepsilon(S) \geq \varepsilon(\mathcal{T})>2 / 3$. Firstly, if $b<a / 2$, then Method I will do. Further, $x /\lceil x\rceil>2 / 3$ for all $x>4 / 3$, so Method III will do when $y>1 / 3$. This leaves only the case $a \leq 2 b$ and $y \leq 1 / 3$. In this case $c=y(a+b) \leq(a+b) / 3 \leq b$, so Method II applies, and yields

$$
\varepsilon(S) \geq \frac{a+2 b+c}{2(a+b)}>\frac{a+2 b}{2 a+2 b}=1-\frac{a}{2 a+2 b} \geq 2 / 3,
$$


using again $2 b \geq a$. This proves that $\varepsilon(S)>2 / 3$ in all cases, which proves (i).

For (ii), we specialize to $a=b$. We shall need yet another covering algorithm.

Method $I V$. Let $z:=c / a$ and let $m:=\lceil z\rceil \geq 1$. Consider first the translates $\{S+i a: i=0, \ldots, m\}$. It is easy to see that $S^{\prime}:=\bigcup_{i=0}^{m}(S+i a)$ is an interval of length $a+c+(m+1) a=(m+2) a+c$. We may thus cover $\mathbb{R}$ by $\mathcal{T}:=\{S+i a+j((m+2) a+c): i=0, \ldots, m, j \in \mathbb{Z}\}$. This covering is periodic, with period $(m+2) a+c$, and $m+1$ translates of $S$ begin in each period. Hence $\tau(\mathcal{T})=(m+1) /((m+2) a+c)$ and the efficiency is

$$
\begin{aligned}
\varepsilon(S) & \geq \varepsilon(\mathcal{T})=\frac{1}{\tau(\mathcal{T}) \lambda(S)}=\frac{(m+2) a+c}{2(m+1) a}=\frac{m+2+z}{2 m+2}=\frac{m+2+z}{\lceil m+2+z\rceil} \\
& >3 / 4,
\end{aligned}
$$

since $m \geq 1$ and $x /\lceil x\rceil>3 / 4$ for all $x>3$.

We next give two examples showing that the bounds given in Theorem 10.1 are indeed best possible.

Example 10.2. Let $S=[0,2] \cup[3+\delta, 4+\delta]$ for $\delta>0$; thus $\lambda(S)=3$. Obviously, we can ignore the shorter interval in $S$ and tile $\mathbb{R}$ by translates of the larger one; this shows that $\tau(S) \leq 1 / 2$ and so $\varepsilon(S)=1 /(3 \tau(S)) \geq 2 / 3$. Theorem 10.1 shows that we can do a little better (for example using method (iii) in the proof above), i.e., that $\varepsilon(S)>2 / 3$. However, as we shall now see, for $\delta$ small we cannot do much better: the shorter interval is essentially useless.

Suppose that $\left\{t_{i}+S\right\}_{i=1}^{N}$ is a covering of a (long) interval $[0, x]$. We may assume that $t_{1}<t_{2}<\cdots<t_{N}$. Let $k_{0}=\inf \left\{k: t_{k} \geq 0\right\}$, and let $k_{1}=\sup \left\{k: t_{k}<x-(4+\delta)\right\}$.

Suppose that $k_{0} \leq k \leq k_{1}$. Then the 'gap' $I=\left(t_{k}+2, t_{k}+3+\delta\right)$ in $t_{k}+S$ lies within $[0, x]$, and is thus covered by the sets $t_{i}+S$.

We consider three cases. Suppose first that $I$ is covered entirely by 'later' translates, i.e., sets of the form $t_{i}+S$ with $i>k$. Then the left endpoint of $I$ lies in the first interval $t_{i}+[0,2]$ of some such $t_{i}+S$, so we have

$$
t_{k+1} \leq t_{i} \leq t_{k}+2 .
$$

Suppose next that $I$ is covered partly by some $t_{i}+S, i<k$, and partly by some $t_{j}+S, j>k$. Then two such sets must meet, and

$$
t_{k+1}-t_{k-1} \leq t_{j}-t_{i} \leq 4+\delta .
$$

Finally, suppose that $I$ is covered entirely by sets $t_{i}+S, i<k$. In any such set, only the shorter interval can meet $I$, so it takes at least two such sets $t_{i}+S, t_{j}+S$ to cover $I$, and assuming that $i<j$ we have

$$
t_{k-2} \geq t_{i} \geq\left(t_{k}+2\right)-(4+\delta)
$$


so $t_{k} \leq t_{k-2}+2+\delta$. But we always have $t_{k+1} \leq t_{k}+\sup S-\inf S=t_{k}+4+\delta$, so in this case

$$
t_{k+1} \leq t_{k-2}+6+2 \delta .
$$

Combining (10.4), (10.5) and (10.6), we see that in all cases there is some $1 \leq i \leq 3$ such that $t_{k+1} \leq t_{k+1-i}+i(2+\delta)$, and it follows by induction that $t_{k} \leq(2+\delta)\left(k-k_{0}+2\right)$ for all $k \in\left[k_{0}-2, k_{1}+1\right]$. Since $t_{k_{1}+1} \geq x-(4+\delta)=x-O(1)$, it follows that the number $N$ of translates satisfies $N \geq k_{1}-k_{0}+1 \geq x /(2+\delta)-O(1)$. Since $x$ was arbitrary we have $\tau(S) \geq 1 /(2+\delta)$ and $\varepsilon(S)=1 /(\lambda(S) \tau(S)) \leq(2+\delta) / 3$.

The next example shows that for a union $S$ of two intervals of equal lengths, the bound $\varepsilon(S)>3 / 4$ given by Theorem 10.1 is best possible.

Example 10.3. Let $S=[0,1] \cup[1+\delta, 2+\delta]$ where $\delta>0$. Suppose that $S_{1}, \ldots, S_{N}$ are translates of $S$ covering $I=[0, x]$, and hence covering $I^{\prime}=$ $[2+\delta, x-(2+\delta)]$. For $y \in I^{\prime}$ let $f(y)$ be the number of times $y$ is covered, so $f(y)=\sum_{i} \chi_{S_{i}}(y)$, where $\chi_{A}$ denotes the characteristic function of a set $A$.

Set

$$
w\left(S_{i}\right)=\int_{S_{i} \cap I^{\prime}} \frac{\mathrm{d} y}{f(y)}=\int_{I^{\prime}} \frac{\chi_{S_{i}}(y)}{f(y)} \mathrm{d} y .
$$

Note that $w\left(S_{i}\right)$ may be thought of as the amount of 'covering work' done by $S_{i}$, where the work in covering a point multiple times is split between the sets covering it. Then

$$
\sum_{i=1}^{N} w\left(S_{i}\right)=\int_{I^{\prime}} \frac{\sum_{i} \chi_{S_{i}}(y)}{f(y)} \mathrm{d} y=\int_{I^{\prime}} 1 \mathrm{~d} y=x-4-2 \delta .
$$

Suppose that $w\left(S_{i}\right)>0$, so $S_{i}$ meets $I^{\prime}$ and hence $S_{i}$ lies entirely within $[0, x]$. Let $I_{i}$ be the gap of length $\delta$ in $S_{i}$. Then $I_{i}$ is covered by $\bigcup_{j \neq i} S_{j}$. In particular, some $S_{j}$ meets $I_{i}$. But then $S_{j}$ contains a unit interval $J$ meeting $I_{i}$. Then $J \subset S_{i} \cup I_{i}$, so $\lambda\left(S_{j} \cap S_{i}\right) \geq \lambda\left(J \cap S_{i}\right)=1-\lambda\left(J \cap I_{i}\right) \geq 1-\delta$. Since $f(y) \geq 2$ on $S_{j} \cap S_{i} \cap I^{\prime}$, we have $\chi_{I^{\prime}}(y) / f(y) \leq 1 / 2$ on a subset of $S_{i}$ of measure at least $1-\delta$. Since $\chi_{I^{\prime}}(y) / f(y) \leq 1$ on all of $S_{i}$, it follows that

$$
w\left(S_{i}\right)=\int_{S_{i}} \frac{\chi_{I^{\prime}}(y)}{f(y)} \mathrm{d} y \leq \lambda\left(S_{i}\right)-(1-\delta) / 2=(3+\delta) / 2 .
$$

Since $S_{i}$ was any translate with $w\left(S_{i}\right)>0$, referring back to (10.7) we see that $N(3+\delta) / 2 \geq(x-4-2 \delta)=x-O(1)$. Since $x$ was arbitrary, it follows that $\tau(S) \geq 2 /(3+\delta)$, so $\varepsilon(S)=1 /(\tau(S) \lambda(S)) \leq(3+\delta) / 4$.

Together, Theorem 10.1 and Examples 10.2 and 10.3 establish the result we promised earlier.

Corollary 10.4. For $k=2$ the quantities defined in (8.6) and (8.7) satisfy $\beta_{2}=\frac{2}{3}$ and $\gamma_{2}=\frac{3}{4}$. 


$$
\text { 11. } G=\mathbb{Z}^{d}
$$

In this and the next section we briefly consider coverings of higher dimensional spaces, starting with $\mathbb{Z}^{d}$. In dimensions two and higher the notion of the density of a general set $T \subset \mathbb{Z}^{d}$ is a little slippery: it is naturally defined as the limit (if it exists) of the fraction of a large 'ball' that lies in $T$, but the existence and value of the limit may depend on the norm chosen to define the balls. This ambiguity does not arise for periodic sets $T$, i.e., sets invariant under translation through the elements of some lattice $\mathcal{L} \subset \mathbb{Z}^{d}$. If $S$ is finite and $T+S=\mathbb{Z}^{d}$, then for any reasonable notion of density there are periodic sets $T^{\prime}$ with density arbitrarily close to that of $T$ such that $T^{\prime}+S=\mathbb{Z}^{d}$, so the covering density of a finite set $S$ turns out to make very good sense. Rather than discuss this further, we simply pick one explicit definition.

Given a finite, non-empty subset $S$ of $\mathbb{Z}^{d}, d \geq 2$, let $\tau\left(S,[n]^{d}\right)$ be the smallest number of translates of $S$ that cover the cube $[n]^{d}$. For $n$ large (greater than the maximum difference between corresponding coordinates of points in $S$ ) we may regard $S$ as a subset of the discrete torus $\mathbb{Z}_{n}^{d}$. Then (6.1) extends to $\tau\left(S, \mathbb{Z}_{n}^{d}\right) \leq \tau\left(S,[n]^{d}\right)$. Moreover, the proof of Lemma 6.1] is easily modified to give the following result.

Lemma 11.1. If $S \subset \mathbb{Z}^{d}$ is finite and non-empty, then the limits

$$
\lim _{n \rightarrow \infty} \frac{\tau\left(S,[n]^{d}\right)}{n^{d}} \quad \text { and } \quad \lim _{n \rightarrow \infty} \frac{\tau\left(S, \mathbb{Z}_{n}^{d}\right)}{n^{d}}
$$

exist and are equal.

The covering density $\tau(S)$ of $S$ is defined to be the common value of the limits above and, as in Section 5, the efficiency of $S$ is

$$
\varepsilon(S):=1 /(\tau(S)|S|)=\lim _{n \rightarrow \infty} \varepsilon\left(S, \mathbb{Z}_{n}^{d}\right) .
$$

By (3.6), if $|S|=k$ then $\tau(S) \leq H_{k} / k$ and $\varepsilon(S) \geq 1 / H_{k}$, just as for subsets of $\mathbb{Z}$.

One interesting case is when $S$ is a product set: suppose that $S=S_{1} \times S_{2}$ with $S_{1} \subset \mathbb{Z}^{d_{1}}$ and $S_{2} \subset \mathbb{Z}^{d_{2}}$. Then (11.1) and Lemma 3.6] show that

$$
\varepsilon\left(S_{1}\right) \varepsilon\left(S_{2}\right) \leq \varepsilon\left(S_{1} \times S_{2}\right) \leq \min \left\{\varepsilon\left(S_{1}\right), \varepsilon\left(S_{2}\right)\right\} .
$$

The argument in Example 4.8 and Theorem 6.6 show that the first inequality may be strict.

Turning to sets of a given size, let $\alpha_{k, d}=\inf \left\{\varepsilon(S): S \subset \mathbb{Z}^{d},|S|=k\right\}$. Taking $S_{2}=\{0\}$ in (11.2), it follows that $\alpha_{k, 1} \geq \alpha_{k, 2} \geq \cdots$.

Question 11.2. Is $\alpha_{k, d}$ equal to $\alpha_{k}\left(=\alpha_{k, 1}\right)$ for $d>1$ ?

Note that the lower bound $\alpha_{k, d} \geq 1 / H_{k}$ holds for every $d$, as a consequence of (3.8) and (11.1).

In general, subsets of $\mathbb{Z}^{d}$ seem much harder to handle than subsets of $\mathbb{Z}$. For example, unlike in the case $d=1$ discussed in Remark 5.4, we do not 
know of an algorithm that given as input a finite set $S \subset \mathbb{Z}^{d}$ calculates $\varepsilon(S)$; indeed, it may well be the case that no such algorithm exists. If we consider a finite set $S_{1}, \ldots, S_{k}$ of finite subsets of $\mathbb{Z}^{d}$, and simply ask whether there is a partition of $\mathbb{Z}^{d}$ into sets each of which is a translate of some $S_{i}$, then this a form of the classical domino tiling problem discussed by Wang [53]. Usually, one considers square tiles with coloured edges, and asks whether $\mathbb{Z}^{2}$ can be tiled by translates of a given set of such Wang tiles so that the colours on adjacent edges match; it is easy to code any problem of this form with suitable sets $S_{i}$. As shown by Berger [7], this tiling problem is undecidable; a key related fact is the existence of sets of tiles which do tile $\mathbb{Z}^{2}$, but not in a periodic way.

Returning to our problem, it may be that the question of deciding whether a given $S$ has a covering of a certain efficiency is undecidable. Moreover, it seems to be still open to determine whether there is always an optimal periodic covering, or indeed whether $\varepsilon(S)$ is necessarily rational. In fact, even for the special case of tilings, i.e., sets with $\varepsilon(S)=1$, the corresponding questions seem to be still open; see, for example, Rao and Xue 44]. There are some partial results: for example, Wijshoff and van Leeuwen [55] showed that if $S \subset \mathbb{Z}^{2}$ is connected and contains no 'holes' (so $S$ corresponds to a polyomino), then $S$ tiles $\mathbb{Z}^{2}$ if and only if it does so in a periodic manner, and gave an algorithm to determine whether such a tiling exists (see also Beauquier and Nivat [6]). In a different direction, Schmidt and Tuller [49] give a criterion for deciding whether a set $S$ with $|S|=4$ tiles $\mathbb{Z}^{d}$.

\section{2. $G=\mathbb{R}^{d}$}

Finally, one can also consider subsets $S$ of $\mathbb{R}^{d}$, in the same way as in Section 8 . Here, many classes of subsets seem natural to consider. For example, as noted in the introduction, the case when $S$ is a ball is a classical problem, and the case where $S \subset \mathbb{R}^{2}$ is convex polygon has also received much attention; see $[17 ; 18 ; 45]$. As in the previous section, there is an apparent difficulty defining the covering density of $S$, but for the bounded sets $S$ we consider, one need only consider periodic coverings, and the problem disappears; we omit the details.

Another interesting case, generalizing that discussed in Section 9, is when $S \subset \mathbb{R}^{2}$ is a union of $k$ rectangles $R_{i}$ of the form $R_{i}=I_{i} \times J_{i}$, which we may or may not insist are disjoint. (Of course, this extends to $d \geq 3$.) The discretization argument used in the proof of Theorem 9.1 does not work in this context: even if they have the same area, the rectangles may have very different aspect ratios, and there may be no 'grid' that approximates all of them well. In fact, we do not know any lower bound for the efficiency $\varepsilon(S)$ better than the trivial bound $\varepsilon(S) \geq 1 / k$, obtained by ignoring all rectangles in $S$ except one with maximal area. 
Question 12.1. Writing $\beta_{k, d}$ for the infimum of $\varepsilon(S)$ over all sets $S \subset \mathbb{R}^{d}$ that are unions of $k$ axis-aligned rectangles, do we have $\beta_{k, d}=1 / k$ for all (or any) $k \geq 2$ and $d \geq 2$ ?

In dimension two, one natural candidate for a very inefficient set $S$ with $k=2$ is the 'thin cross' $S_{L}=([-L, L] \times[-1,1]) \cup([-1,1] \times[-L, L])$ with $L$ large. However, as noted by Everett and Hickerson [14] (whose main purpose in that paper was to relate suitable coverings of $\mathbb{R}^{d}$ to corresponding ones of $\left.\mathbb{Z}^{d}\right), \varepsilon\left(S_{L}\right) \geq 3 / 4+o(1)$ as $L \rightarrow \infty$. More precisely, they conjectured that when $L=2 a+1$ with $a$ an integer (so $S_{L}$ is the union of $4 a+1$ congruent squares), then $\kappa\left(S_{L}\right)=(4 a+1) /(3 a+2)$, and proved that $\kappa\left(S_{L}\right)$ is at most this large by giving an example of a covering. Loomis [34] proved their conjecture for $a=2$, but in general it seems to be still open. For a survey of related questions concerning crosses and 'semi-crosses' in various dimensions, see Stein [50].

Perhaps a better candidate for the union $S$ of two rectangles with $\varepsilon(S)$ small is the set $S_{L}^{\prime}=([1, L] \times[0,1]) \cup([0,1] \times[1, L])$ formed by two arms of a cross without the central square. In this case, for large $L$ we do not have a better lower bound on $\varepsilon\left(S_{L}^{\prime}\right)$ than the trivial $\varepsilon\left(S_{L}^{\prime}\right) \geq 1 / 2$.

We are so far from knowing the answer to Question 12.1 above that we do not even know whether $k \beta_{k, 2}$ is bounded as $k \rightarrow \infty$. One plausible approach to proving that it is bounded is to consider sets $S$ formed as the union of $k$ rectangles with a common centre and unit area, but with very different aspect ratios. But even in this case we do not know the covering efficiency; it may be that even if the aspect ratios form a rapidly increasing sequence, $\varepsilon(S)$ is larger than $1 / k$ by more than a constant factor as $k \rightarrow \infty$. If so, then, fitting $S$ inside the region $H$ bounded by the hyperbola $|x||y|=1$, it would follow that for any $\varepsilon>0$ there is a periodic covering $T+H$ of $\mathbb{R}^{2}$ by hyperbolic regions in which $T$ has (upper) density at most $\varepsilon$. (It is not hard to construct an unrestricted covering in which the density of $T$, defined as the limit of $\left(\pi r^{2}\right)^{-1}$ times the number of points within a disk of radius $r$, is zero.) The question of whether such periodic coverings exists seems of interest in its own right; it has the flavour of number theory/approximation theory, and may well be known, but we have not found a reference.

Of course, one can also consider many other families of sets, such as unions of rectangles with arbitrary orientations. In general, it seems that most questions one can ask in dimension two or higher are rather difficult.

\section{APPENDiX A.}

In this appendix we prove Lemma 7.7, and show that Theorem 7.1 follows. Our proof of Theorem 7.1 is simpler in the case when $n$ is prime, so we begin with this case (which suffices to prove Corollary 7.5) and return to the general case later.

To handle the case where $n$ is prime, the following weaker form of Lemma 7.7 suffices; the only difference is that we have omitted the condition $(z, n)=$ 
1 from part (ii). Recall that $\bar{x}$ denotes the element in $\mathbb{Z}_{n}$ corresponding to an integer $x \in \mathbb{Z}$.

Lemma A.1. For every $m \geq 1$ and $\delta>0$ there exists $C=C(m, \delta)$ such that for every $n$ and every $b_{1}, \ldots, b_{m} \in \mathbb{Z}$, if $\bar{x}_{1}, \ldots, \bar{x}_{m}$ are any $m$ elements of $\mathbb{Z}_{n}$, then at least one of the following holds.

(i) There exists a vanishing linear combination $\sum_{i=1}^{m} a_{i} \bar{x}_{i}=0$ in $\mathbb{Z}_{n}$ with integer coefficients $a_{1}, \ldots, a_{m}$ satisfying $0<\max _{i}\left|a_{i}\right| \leq C$.

(ii) There exist $z \in \mathbb{Z}$ and $y_{1}, \ldots, y_{m} \in[0, \delta n] \cap \mathbb{Z}$ such that $z \bar{x}_{i}-\bar{b}_{i}=\bar{y}_{i}$ in $\mathbb{Z}_{n}$, i.e.,

$$
z x_{i}-b_{i} \equiv y_{i} \quad(\bmod n), \quad i=1, \ldots, m .
$$

Proof. We use Fourier analysis. Set $\omega:=\exp (2 \pi \mathrm{i} / n)$, and define the Fourier transform of a function $f: \mathbb{Z}_{n} \rightarrow \mathbb{C}$ by

$$
\widehat{f}(\bar{a})=\sum_{\bar{x} \in \mathbb{Z}_{n}} f(\bar{x}) \omega^{a x}, \quad a \in \mathbb{Z} .
$$

If $\delta \geq 1$ then (ii) holds trivially for any $z$. Provided we choose $C \geq 4 / \delta$, as we may, if $n \leq 4 / \delta$ then (i) holds trivially with all $a_{i}$ equal to $n$. Hence we may assume that $\delta<1$ and $n>4 / \delta$.

Let $\ell:=\lfloor\delta n / 2\rfloor$ and $I:=\{0, \ldots, \ell\} \subset \mathbb{Z}_{n}$. Define $g: \mathbb{Z}_{n} \rightarrow[0, \infty)$ by $g:=n^{-2} \chi_{I} * \chi_{I}$, so

$$
g(\bar{x})=n^{-2} \sum_{\bar{y} \in \mathbb{Z}_{n}} \chi_{I}(\bar{x}-\bar{y}) \chi_{I}(\bar{y}) .
$$

For $i=1, \ldots, m$, define $h_{i}$ by setting $h_{i}(\bar{x})=g\left(\bar{x}-\bar{b}_{i}\right)$. Note that

$$
\{x \in\{0, \ldots, n-1\}: g(\bar{x}) \neq 0\} \subseteq[0,2 \ell] \subseteq[0, \delta n] .
$$

For every $a \in \mathbb{Z}$,

$$
\left|\widehat{\chi_{I}}(\bar{a})\right|=\left|\sum_{x=0}^{\ell} \omega^{a x}\right|=\left|\frac{1-\omega^{a(\ell+1)}}{1-\omega^{a}}\right| \leq \frac{2}{\left|1-\omega^{a}\right|}=\frac{1}{|\sin (\pi a / n)|} .
$$

Also,

$$
\left|\widehat{\chi_{I}}(\bar{a})\right|=\left|\sum_{x=0}^{\ell} \omega^{a x}\right| \leq \ell+1 \leq n .
$$

For all $a \in \mathbb{Z}$ with $|a| \leq n / 2$ we have $|\sin (\pi a / n)| \geq 2|a| / n$, and hence

$$
\left|\widehat{\chi_{I}}(\bar{a})\right| \leq \min \left(n, \frac{n}{2|a|}\right) \leq \frac{n}{1+|a|} .
$$

Hence, for $|a| \leq n / 2$,

$$
\left|\widehat{h_{i}}(\bar{a})\right|=|\widehat{g}(\bar{a})|=\left|n^{-2} \widehat{\chi_{I}}(\bar{a})^{2}\right| \leq \frac{1}{(1+|a|)^{2}} .
$$

Moreover,

$$
\widehat{h_{i}}(0)=\widehat{g}(0)=n^{-2} \widehat{\chi_{I}}(0)^{2}=n^{-2}(\ell+1)^{2} \geq(\delta / 2)^{2} .
$$


Now define $H: \mathbb{Z}_{n}^{m} \rightarrow \mathbb{R}$ as the tensor product of the $h_{i}$, so

$$
H\left(\bar{x}_{1}, \ldots, \bar{x}_{m}\right):=\prod_{i=1}^{m} h_{i}\left(\bar{x}_{i}\right) .
$$

By (A.2), when $\left|a_{i}\right| \leq n / 2$ for all $i$,

$$
\left|\widehat{H}\left(\bar{a}_{1}, \ldots, \bar{a}_{m}\right)\right|=\left|\prod_{i=1}^{m} \widehat{h}_{i}\left(\bar{a}_{i}\right)\right| \leq \prod_{i=1}^{m}\left(1+\left|a_{i}\right|\right)^{-2} .
$$

Now fix $\bar{x}_{1}, \ldots, \bar{x}_{m} \in \mathbb{Z}_{n}$, and consider the sum

$$
W:=\sum_{z \in \mathbb{Z}_{n}} H\left(z \bar{x}_{1}, \ldots, z \bar{x}_{m}\right)
$$

where we abuse notation by writing $\mathbb{Z}_{n}$ for an arbitrary subset of $\mathbb{Z}$ consisting of one element from each residue class modulo $n$.

If this sum does not vanish, then at least one term is non-zero, which means that $h_{i}\left(z \bar{x}_{i}\right) \neq 0$ for some $z$ and all $i$. Fix such a $z$ and define $y_{i}$ by $0 \leq y_{i}<n$ and $\bar{y}_{i}=z \bar{x}_{i}-\bar{b}_{i}$. Then $g\left(\bar{y}_{i}\right)=g\left(z \bar{x}_{i}-\bar{b}_{i}\right)=h_{i}\left(z \bar{x}_{i}\right) \neq 0$. Hence, recalling (A.1), we have $0 \leq y_{i} \leq \delta n$. Consequently (ii) holds whenever $W \neq 0$.

We compute $W$ using Fourier inversion:

$$
\begin{aligned}
n^{m} W & =\sum_{z \in \mathbb{Z}_{n}} \sum_{\bar{a}_{1}, \ldots, \bar{a}_{m} \in \mathbb{Z}_{n}} \widehat{H}\left(\bar{a}_{1}, \ldots, \bar{a}_{m}\right) \omega^{-\sum_{j=1}^{m} z x_{j} a_{j}} \\
& =\sum_{\bar{a}_{1}, \ldots, \bar{a}_{m} \in \mathbb{Z}_{n}} \widehat{H}\left(\bar{a}_{1}, \ldots, \bar{a}_{m}\right) \sum_{z \in \mathbb{Z}_{n}} \omega^{-z \sum_{j=1}^{m} x_{j} a_{j}} .
\end{aligned}
$$

The inner sum evaluates to $n$ if $\sum_{j=1}^{m} x_{j} a_{j} \equiv 0(\bmod n)$, and vanishes otherwise. Hence, representing $\mathbb{Z}_{n}$ by the interval $J_{n}:=\{z \in \mathbb{Z}:-n / 2<z \leq$ $n / 2\}$,

$$
n^{m-1} W=\sum_{\left(\bar{a}_{1}, \ldots, \bar{a}_{m}\right) \in E} \widehat{H}\left(\bar{a}_{1}, \ldots, \bar{a}_{m}\right),
$$

where

$$
E:=\left\{\left(a_{1}, \ldots, a_{m}\right) \in J_{n}^{m}: \sum_{j=1}^{m} a_{j} \bar{x}_{j}=0 \text { in } \mathbb{Z}_{n}\right\} .
$$

Clearly, $(0, \ldots, 0) \in E$, and, by (A.3),

$$
\widehat{H}(0, \ldots, 0)=\prod_{i=1}^{m} \widehat{h}_{i}(0)=\widehat{g}(0)^{m} \geq(\delta / 2)^{2 m} .
$$


If (i) does not hold, then $(E \backslash\{(0, \ldots, 0)\}) \cap[-C, C]^{m}=\emptyset$, and thus (A.8), (A.9) and (A.5) yield

$$
\begin{aligned}
n^{m-1} W & \geq \prod_{i=1}^{m} \widehat{h}_{i}(0)-\sum_{E \backslash\{(0, \ldots, 0)\}}\left|\widehat{H}\left(\bar{a}_{1}, \ldots, \bar{a}_{m}\right)\right| \\
& \geq\left(\frac{\delta}{2}\right)^{2 m}-\sum_{\mathbb{Z}^{m} \backslash[-C, C]^{m}} \prod_{i=1}^{m}\left(1+\left|a_{i}\right|\right)^{-2} .
\end{aligned}
$$

Since

$$
\sum_{\mathbb{Z}^{m}} \prod_{i=1}^{m}\left(1+\left|a_{i}\right|\right)^{-2}=\left(\sum_{a=-\infty}^{\infty}(1+|a|)^{-2}\right)^{m}<\infty,
$$

we can choose $C=C(m, \delta)$ such that the last sum in (A.10) is less that $(\delta / 2)^{2 m}$, and then (A.10) implies $W>0$.

In summary, choosing $C$ as above, if (i) does not hold, then $W \neq 0$, which we have shown implies (ii).

We can now prove our weak version of Theorem 7.1.

Theorem A.2. Theorem 7.1 holds if the conclusion is restricted to prime values of $n$.

Proof. We may assume that $\varepsilon \leq 1 / 2$. Provided we choose $C \geq 2 k / \varepsilon$, as we may, if $n \leq 2 k / \varepsilon$ then (7.1) holds with $a_{1}=-a_{2}=n$ and the other $a_{i}$ equal to 0 . We may thus assume that $n>2 k / \varepsilon$ (and so $n>8$ ).

Both (7.1) and $\kappa\left(S, \mathbb{Z}_{n}\right)$ are preserved by adding a constant to all elements of $S$, so we may and shall assume that $\bar{x}_{k}=0$. We apply Lemma A.1 with $m:=k-1, \delta:=\varepsilon /(2 k)$ and $b_{i}:=\lceil i n / k\rceil$.

Suppose first that Lemma A.1(i) holds. Then $\sum_{i=1}^{m} a_{i} \bar{x}_{i}=0$ for some $a_{i}$ with $0<\max _{i}\left|a_{i}\right| \leq C$. Set $a_{k}=-\sum_{i=1}^{m} a_{i}$; then, recalling that $\bar{x}_{k}=0$, we have $\sum_{i=1}^{k} a_{i} \bar{x}_{i}=0, \sum_{i=1}^{k} a_{i}=0$ and $\left|a_{i}\right| \leq m C$, so our conclusion (7.1) holds (with a different $C$ ).

Suppose instead that Lemma A.1(ii) holds. Then, for some $z \in \mathbb{Z}$ we have $z x_{i} \equiv x_{i}^{\prime}(\bmod n)$ for $i=1, \ldots, m=k-1$, where $x_{i}^{\prime}:=b_{i}+y_{i} \in\left[b_{i}, b_{i}+\delta n\right]$. Now

$$
\frac{i n}{k} \leq x_{i}^{\prime}<\left(\frac{i}{k}+\delta\right) n+1 \text {. }
$$

Furthermore, since $\bar{x}_{k}=0$, we have $z x_{k} \equiv 0(\bmod n)$. Let $x_{0}^{\prime}:=0$ and $x_{k}^{\prime}:=n$. For $1 \leq i \leq k$, by (A.12) the gap $x_{i}^{\prime}-x_{i-1}^{\prime}$ is less than $(1 / k+\delta) n+1$. Hence, if $S^{\prime}:=\left\{\bar{x}_{i}^{\prime}: i=1, \ldots, k\right\} \subseteq \mathbb{Z}_{n}$ and $T:=\{0,1, \ldots,\lfloor(1 / k+\delta) n\rfloor\}$, then $T+S^{\prime}=\mathbb{Z}_{n}$. Consequently,

$$
\tau\left(S^{\prime}, \mathbb{Z}_{n}\right) \leq|T| \leq\left(\frac{1}{k}+\delta\right) n+1
$$

and thus

$$
\kappa\left(S^{\prime}, \mathbb{Z}_{n}\right)=\frac{k}{n} \tau\left(S^{\prime}, \mathbb{Z}_{n}\right) \leq 1+\delta k+k / n<1+\varepsilon
$$


Since $1 / k+\delta \leq \frac{1}{2}+\frac{1}{8}<\frac{2}{3}$, A.12 then implies $0<x_{1}^{\prime}<2 n / 3+1<n$ and thus $z x_{i} \equiv x_{1}^{\prime} \not \equiv 0(\bmod n)$; hence, $z \not \equiv 0(\bmod n)$. Using the simplifying assumption that $n$ is prime, the map $\bar{x} \mapsto z \bar{x}$ is an automorphism of $\mathbb{Z}_{n}$ onto itself. Hence,

$$
\kappa\left(S, \mathbb{Z}_{n}\right)=\kappa\left(z S, \mathbb{Z}_{n}\right)=\kappa\left(S^{\prime}, \mathbb{Z}_{n}\right)<1+\varepsilon,
$$

contradicting our assumptions and completing the proof.

To prove Theorem 7.1, we need Lemma 7.7 in place of Lemma A.1. Recall that the only difference is that in conclusion (ii), the former allows us to assume that the greatest common divisor $(z, n)$ of $z$ and $n$ is equal to 1 . Before proving Lemma [7.7, let us first show that Theorem 7.1 follows.

Proof of Theorem 7.1. The proof is the same as the proof of Theorem A.2 given above, except that we use Lemma 7.7 instead of Lemma A.1. In the case where Lemma 7.7(ii) holds we have $(z, n)=1$, so $\bar{x} \mapsto z \bar{x}$ is still an automorphism of $\mathbb{Z}_{n}$ even though $n$ need not be prime.

Let $\mathbb{Z}_{n}^{*}:=\left\{x \in \mathbb{Z}_{n}:(x, n)=1\right\}$ (again regarded as a subset of $\mathbb{Z}$ when convenient), and let $\phi(n):=\left|\mathbb{Z}_{n}^{*}\right|$, be the Euler phi function, i.e., the number of integers $1 \leq x<n$ that are coprime to $n$. Let $\chi_{n}^{*}:=\chi_{\mathbb{Z}_{n}^{*}}$ denote the characteristic function of $\mathbb{Z}_{n}^{*} \subset \mathbb{Z}_{n}$.

It remains to prove Lemma 7.7, we shall deduce Lemma 7.7 from the following lemma, whose proof we in turn postpone.

Lemma A.3. Given $\eta>0$, let $\mathcal{P}:=\{p$ prime and $p \leq 1+1 / \eta\}$ and let $P:=\prod_{p \in \mathcal{P}} p$. Then, for every $n \geq 1$ and $u \in \mathbb{Z}$, either $P u \equiv 0(\bmod n)$ or $\left|\widehat{\chi_{n}^{*}}(\bar{u})\right|<\eta \phi(n)$.

Proof of Lemma 7.7. Define $H$ as in the proof of Lemma A.1, but consider now

$$
W^{*}:=\sum_{z \in \mathbb{Z}_{n}^{*}} H\left(z \bar{x}_{1}, \ldots, z \bar{x}_{m}\right) .
$$

As before, if $W^{*} \neq 0$, then (ii) (in its new stronger version) holds.

We have, in analogy with (A.7),

$$
\begin{aligned}
n^{m} W^{*} & =\sum_{\bar{a}_{1}, \ldots, \bar{a}_{m} \in \mathbb{Z}_{n}} \widehat{H}\left(\bar{a}_{1}, \ldots, \bar{a}_{m}\right) \sum_{z \in \mathbb{Z}_{n}^{*}} \omega^{-z \sum_{j=1}^{m} x_{j} a_{j}} \\
& =\sum_{\bar{a}_{1}, \ldots, \bar{a}_{m} \in \mathbb{Z}_{n}} \widehat{H}\left(\bar{a}_{1}, \ldots, \bar{a}_{m}\right) \widehat{\chi_{n}^{*}}\left(-\sum_{j=1}^{m} a_{j} \bar{x}_{j}\right) .
\end{aligned}
$$

Let $M\left(a_{1}, \ldots, a_{m}\right):=\prod_{i=1}^{m}\left(1+a_{i}^{2}\right)^{-1}$, so that (A.5) becomes

$$
\left|\widehat{H}\left(\bar{a}_{1}, \ldots, \bar{a}_{m}\right)\right| \leq M\left(a_{1}, \ldots, a_{m}\right) .
$$


Note that $\sum_{\mathbb{Z}^{m}} M\left(a_{1}, \ldots, a_{m}\right)<\infty$ by (A.11), so we may choose $C_{1}$ and $\eta>0$ such that

$$
\sum_{\mathbb{Z}^{m} \backslash\left[-C_{1}, C_{1}\right]^{m}} M\left(a_{1}, \ldots, a_{m}\right)<\frac{1}{2}\left(\frac{\delta}{2}\right)^{2 m}
$$

and

$$
\eta \sum_{\mathbb{Z}^{m}} M\left(a_{1}, \ldots, a_{m}\right)<\frac{1}{2}\left(\frac{\delta}{2}\right)^{2 m} .
$$

Suppose first that

$$
\left|\widehat{\chi_{n}^{*}}\left(-\sum_{j=1}^{m} a_{j} \bar{x}_{j}\right)\right|>\eta \phi(n)
$$

for some $\left(a_{1}, \ldots, a_{m}\right) \in\left[-C_{1}, C_{1}\right]^{m} \backslash\{(0, \ldots, 0)\}$. Then, by Lemma A.3, for a certain integer $P$ depending only on $\eta$, we have $\sum_{i=1}^{m} P a_{i} x_{i} \equiv 0(\bmod n)$, so the conclusion (i) of Lemma 7.7 holds with $a_{i}$ replaced by $a_{i}^{\prime}:=P a_{i}$ and $C:=P C_{1}$.

On the other hand, if (A.17) fails for all $\left(a_{1}, \ldots, a_{m}\right) \in\left[-C_{1}, C_{1}\right]^{m} \backslash$ $\{(0, \ldots, 0)\}$, then from (A.13), A.14), (A.9), and A.16) we have

$$
\begin{aligned}
n^{m} W^{*} \geq \widehat{H}(0, \ldots, 0) \phi(n)-\sum_{\mathbb{Z}^{m} \backslash\{(0, \ldots, 0)\}} M\left(a_{1}, \ldots, a_{m}\right)\left|\widehat{\chi_{n}^{*}}\left(-\sum_{j=1}^{m} a_{j} \bar{x}_{j}\right)\right| \\
\geq\left(\frac{\delta}{2}\right)^{2 m} \phi(n)-\sum_{\left[-C_{1}, C_{1}\right]^{m} \backslash\{(0, \ldots, 0)\}} M\left(a_{1}, \ldots, a_{m}\right) \eta \phi(n) \\
\quad-\sum_{\mathbb{Z}^{m} \backslash\left[-C_{1}, C_{1}\right]^{m}} M\left(a_{1}, \ldots, a_{m}\right) \phi(n) \\
>\left(\frac{\delta}{2}\right)^{2 m} \phi(n)\left(1-\frac{1}{2}-\frac{1}{2}\right)=0 .
\end{aligned}
$$

Thus $W^{*} \neq 0$, and thus, as shown above, the conclusion (ii) of Lemma 7.7 holds.

It remains to prove our estimate Lemma A.3 of the Fourier transform $\widehat{\chi_{n}^{*}}$ of $\chi_{n}^{*}=\chi_{\mathbb{Z}_{n}^{*}}$. First, we state (and this time immediately prove) a final lemma.

Lemma A.4. Let $n=\prod_{i=1}^{r} p_{i}^{e_{i}}$ be the prime factorization of $n \geq 2$. Then for any $u \in \mathbb{Z}$ we have $\widehat{\chi_{n}^{*}}(\bar{u})=\prod_{i=1}^{r} \psi_{p_{i} e_{i}}(u)$, where

$$
\psi_{p^{e}}(u):= \begin{cases}0, & p^{e-1} \nmid u ; \\ -p^{e-1}, & p^{e-1} \mid u, p^{e} \nmid u ; \\ p^{e}-p^{e-1}, & p^{e} \mid u .\end{cases}
$$


Proof. Set $f\left(y_{1}, \ldots, y_{r}\right):=\sum_{i=1}^{r} y_{i} n / p_{i}^{e_{i}}$, and define $\bar{f}: \prod_{i=1}^{r} \mathbb{Z}_{p_{i}^{e_{i}}} \rightarrow \mathbb{Z}_{n}$ by $\bar{f}\left(\bar{y}_{1}, \ldots, \bar{y}_{r}\right):=\overline{f\left(y_{1}, \ldots, y_{r}\right)}$. Note that $\bar{f}$ is well defined. Furthermore,

$$
f\left(y_{1}, \ldots, y_{r}\right) \equiv y_{i} n / p_{i}^{e_{i}} \quad \bmod p_{i}^{e_{i}}, \quad i=1, \ldots, r
$$

which implies that $\bar{f}$ is injective and thus a bijection. (In fact, $\bar{f}$ is the map whose existence is guaranteed by the Chinese Remainder Theorem.) Moreover, by (A.18), $p_{i}\left|f\left(y_{1}, \ldots, y_{r}\right) \Longleftrightarrow p_{i}\right| y_{i}$, and thus $\left(f\left(y_{1}, \ldots, y_{r}\right), n\right)=$ $1 \Longleftrightarrow\left(y_{i}, p_{i}^{e_{i}}\right)=1$ for every $i$; consequently, $\bar{f}$ is also a bijection $\prod_{i=1}^{r} \mathbb{Z}_{p_{i}^{e_{i}}}^{*} \rightarrow$ $\mathbb{Z}_{n}^{*}$. Hence,

$$
\begin{aligned}
\widehat{\chi_{n}^{*}}(\bar{u}) & =\sum_{\bar{x} \in \mathbb{Z}_{n}^{*}} \exp (2 \pi \mathrm{i} u x / n) \\
& =\sum_{\bar{y}_{i} \in \mathbb{Z}^{*}{ }_{p_{i}}, i=1, \ldots, r} \exp \left(2 \pi \mathrm{i} u f\left(y_{1}, \ldots, y_{r}\right) / n\right) \\
& =\sum_{\bar{y}_{i} \in \mathbb{Z}^{*} p_{p_{i}}, i=1, \ldots, r} \prod_{i=1}^{r} \exp \left(2 \pi \mathrm{i} u y_{i} / p_{i}^{e_{i}}\right) \\
& =\prod_{i=1}^{r} \sum_{\bar{y} \in \mathbb{Z}^{*} e_{e_{i}}} \exp \left(2 \pi \mathrm{i} u y / p_{i}^{e_{i}}\right) .
\end{aligned}
$$

For any prime $p$ and any $e \geq 1, \mathbb{Z}_{p^{e}}^{*}$ can be represented by $\{y: 1 \leq y \leq$ $\left.p^{e}\right\} \backslash\left\{p z: 1 \leq z \leq p^{e-1}\right\}$, so

$$
\sum_{\bar{y} \in \mathbb{Z}_{p^{e}}^{*}} \exp \left(2 \pi \mathrm{i} u y / p^{e}\right)=\sum_{y=1}^{p^{e}} \exp \left(2 \pi \mathrm{i} u y / p^{e}\right)-\sum_{z=1}^{p^{e-1}} \exp \left(2 \pi \mathrm{i} u z / p^{e-1}\right)=\psi_{p^{e}}(u),
$$

since

$$
\sum_{y=1}^{p^{k}} \exp \left(2 \pi \mathrm{i} u y / p^{k}\right)= \begin{cases}p^{k}, & p^{k} \mid u \\ 0, & p^{k} \nmid u\end{cases}
$$

for any $k \geq 0$.

Lemma A.3 now follows easily.

Proof of Lemma A.3. Using the notation of LemmaA.4 we see that if $p_{i}^{e_{i}} \nmid u$ for some prime factor $p_{i} \notin \mathcal{P}$ of $n$, then, using $\phi(n)=\widehat{\chi_{n}^{*}}(0)=\prod_{i=1}^{r}\left(p_{i}^{e_{i}}-\right.$ $p_{i}^{e_{i}-1}$ ), we have

$$
\frac{\left|\widehat{\chi_{n}^{*}}(\bar{u})\right|}{\phi(n)} \leq \frac{\left|\psi_{p_{i}}{ }_{i}(u)\right|}{p_{i}^{e_{i}}-p_{i}^{e_{i}-1}} \leq \frac{1}{p_{i}-1}<\eta
$$


Assume $\left|\widehat{\chi_{n}^{*}}(\bar{u})\right| \geq \eta \phi(n)$. Then, by (A.19), $p_{i}^{e_{i}} \mid u$ for every $p_{i} \notin \mathcal{P}$. Furthermore, for every $p_{i} \in \mathcal{P}$ we have $p_{i}^{e_{i}-1} \mid u$, since otherwise $\widehat{\chi_{n}^{*}}(\bar{u})=0$ by Lemma A.4. Hence $n \mid P u$.

As noted above, Lemma A.3 was the only missing piece of the puzzle; the proof of Lemma 7.7 is now complete, and hence that of Theorem 7.1. As shown in Section 7. Theorem 7.2 and Corollaries 7.3 and 7.5 follow.

\section{REFERENCES}

[1] M.J. Appel and R.P. Russo, The maximum vertex degree of a graph on uniform points in $[0,1]^{d}$, Adv. in Appl. Probab. 29 (1997), 567-581.

[2] S. Athreya, R. Roy and A. Sarkar, On the coverage of space by random sets, Adv. in Appl. Probab. 36 (2004), 1-18.

[3] P. Balister, B. Bollobás, A. Sarkar and M. Walters, Connectivity of random $k$-nearest-neighbour graphs, Adv. in Appl. Probab. 37 (2005), $1-24$.

[4] R.P. Bambah and C.A. Rogers, Covering the planes with convex sets, J. London Math. Soc. 27 (1952), 304-314.

[5] R.P. Bambah, C.A. Rogers and H. Zassenhaus, On coverings with convex domains, Acta Arith. 9 (1964), 191-207.

[6] D. Beauquier and M. Nivat, On translating one polyomino to tile the plane, Discrete Comput. Geom. 6 (1991), 575-592.

[7] R. Berger, The undecidability of the domino problem, Mem. Amer. Math. Soc. 66 (1966), 72 pp.

[8] A. Biró, Divisibility of integer polynomials and tilings of the integers, Acta Arith. 118 (2005), 117-127.

[9] K. Böröczky Jr., Finite Packing and Covering, Cambridge Tracts in Mathematics 154, Cambridge University Press, Cambridge, 2004. xviii $+380 \mathrm{pp}$.

[10] E.M. Coven and A. Meyerowitz, Tiling the integers with translates of one finite set, J. Algebra 212 (1999), 161-174.

[11] N.G. de Bruijn, A combinatorial problem, Nederl. Akad. Wetensch. 49 (1946), 758-764.

[12] P. Erdős, Some results on additive number theory, Proc. Amer. Math. Soc. 5 (1954) 847-853.

[13] P. Erdős and C.A. Rogers, The covering of $n$-dimensional space by spheres, J. London Math. Soc. 28 (1953), 287-293.

[14] H. Everett and D. Hickerson, Packing and covering by translates of certain nonconvex bodies, Proc. Amer. Math. Soc. 75 (1979), 87-91.

[15] U. Feige, A threshold of $\ln n$ for approximating set cover, J. ACM 45 (1998), 634-652.

[16] G. Fejes Tóth, Packing and covering, in Handbook of Discrete and Computational Geometry, CRC Press Ser. Discrete Math. Appl., 1997, pp. 19-41. 
[17] L. Fejes Tóth, Lagerungen in der Ebene, auf der Kugel und im Raum, Grundlehren der Mathematischen Wissenschaften 65, Springer-Verlag, 1953. $\mathrm{x}+197 \mathrm{pp}$.

[18] L. Fejes Tóth, Lagerungen in der Ebene auf der Kugel und im Raum, Zweite verbesserte und erweiterte Auflage, Grundlehren der mathematischen Wissenschaften 65, Springer-Verlag, 1972. xi+238 pp.

[19] L. Flatto, A limit theorem for random coverings of a circle, Israel J. Math. 15 (1973), 167-184.

[20] I.J. Good, Normal recurring decimals, J. London Math. Soc. 21 (1946), 167-169.

[21] G. Hajós, Sur la factorisation des groupes abéliens, Časopis Pěst. Mat. Fys. 74 (1949), 157-162.

[22] P. Hall, On the coverage of $k$-dimensional space by $k$-dimensional spheres, Ann. Probab. 13 (1985), 991-1002.

[23] P. Hall, Introduction to the Theory of Coverage Processes, Wiley Series in Probability and Mathematical Statistics, 1988. xx+408 pp.

[24] G.H. Hardy and E.M. Wright, An Introduction to the Theory of Numbers. Sixth edition. Revised by D. R. Heath-Brown and J. H. Silverman. Oxford University Press, Oxford, 2008. xxii+621 pp.

[25] S. Janson, Random coverings of the circle with arcs of random lengths, in Probability and Mathematical Statistics, A. Gut and L. Holst, eds., Uppsala Univ., Uppsala (1983), pp. 62-73.

[26] S. Janson, Random coverings in several dimensions, Acta Math. 156 (1986), 83-118.

[27] S. Janson, Random coverings of the circle by arcs with restricted endpoints, J. Appl. Probab. 25 (1988), 215-219.

[28] S. Janson, T. Łuczak and A. Ruciński, Random Graphs. Wiley, New York, 2000.

[29] D.B. Johnson, Efficient algorithms for shortest paths in sparse networks, J. Assoc. Comput. Mach. 24 (1977), 1-13.

[30] D.S. Johnson, Approximation algorithms for combinatorial problems, Proceedings of the Fifth Annual ACM Symposium on the Theory of Computing (Austin, Tex., 1973), J. Comput. System Sci. 9 (1974), 256-278.

[31] R.M. Karp, A characterization of the minimum cycle mean in a digraph, Discrete Math. 23 (1978), 309-311.

[32] S. Konyagin, and I. Łaba, Spectra of certain types of polynomials and tiling of integers with translates of finite sets, J. Number Theory 103 (2003), 267-280.

[33] M. Laczkovich, Orders of absolute measurability, J. Math. Anal. Appl. 251 (2000), 167-186.

[34] P. Loomis, The covering constant for a certain symmetric star body, Österreich. Akad. Wiss. Math.-Natur. Kl. Sitzungsber. II 192 (1983), 295-308. 
[35] G.G. Lorentz, On a problem of additive number theory, Proc. Amer. Math. Soc. 5 (1954), 838-841.

[36] H. Maehara, A threshold for the size of random caps to cover a sphere, Ann. Inst. Statist. Math. 40 (1988), 665-670.

[37] R. Meester and R. Roy, Continuum Percolation, Cambridge Tracts in Mathematics 119, Cambridge University Press, 1996. x+238 pp.

[38] D.J. Newman, Complements of finite sets of integers, Michigan Math. J. 14 (1967), 481-486.

[39] D.J. Newman, Tesselation of integers. J. Number Theory 9 (1977), 107111.

[40] J.B. Orlin and R.K. Ahuja, New scaling algorithms for the assignment and minimum mean cycle problems, Mathematical Programming $\mathbf{5 4}$ (1992), 41-56.

[41] J. Pach and P.K. Agarwal, Combinatorial Geometry, Wiley-Interscience Series in Discrete Mathematics and Optimization, 1995. xiv+354 pp.

[42] M.D. Penrose, On $k$-connectivity for a geometric random graph, Random Structures Algorithms 15 (1999), 145-164.

[43] M. Penrose, Random Geometric Graphs, Oxford Studies in Probability 5, Oxford University Press, 2003, xiv+330 pp.

[44] H. Rao and Y-M. Xue, Tiling $\mathbb{Z}^{2}$ with translations of one set, Discrete Math. Theor. Comput. Sci. 8 (2006), 129-140 (electronic).

[45] C.A. Rogers, Packing and Covering, Cambridge Tracts in Mathematics and Mathematical Physics 54, Cambridge University Press, New York, 1964, viii+111 pp.

[46] V.A. Rohlin, Lectures on the entropy theory of transformations with invariant measure (in Russian), Uspehi Mat. Nauk 22 (1967), 3-56.

[47] W.M. Schmidt, Complementary sets of finite sets, Monatsh. Math 138 (2003), 61-71.

[48] W.M. Schmidt and D.M. Tuller, Covering and packing in $\mathbb{Z}^{n}$ and $\mathbb{R}^{n}$. I, Monatsh. Math. 153 (2008), 265-281.

[49] W.M. Schmidt and D.M. Tuller, Covering and packing in $\mathbb{Z}^{n}$ and $\mathbb{R}^{n}$. II, Monatsh. Math. 160 (2010), 195-210.

[50] S. Stein, Tiling, packing, and covering by clusters, Rocky Mountain J. Math. 16 (1986), 277-321.

[51] F.W. Steutel, Random division of an interval, Statistica Neerlandica 21 (1967), 231-244.

[52] D. Tuller, Some questions in additive number theory. Thesis, Univ. of Colorado, 2002.

[53] H. Wang, Proving theorems by pattern recognition II, Bell System Technical Journal 40 (1961), 1-41.

[54] G. Weinstein, Some covering and packing results in number theory, $J$. Number Theory 8 (1976), 193-205.

[55] H.A.G. Wijshoff and J. van Leeuwen, Arbitrary versus periodic storage schemes and tessellations of the plane using one type of polyomino, Information and Control 62 (1984), 1-25. 
Department of Pure Mathematics and Mathematical Statistics, Wilberforce Road, Cambridge CB3 0WB, UK and Department of Mathematical SciEnCES, University of Memphis, Memphis TN 38152, USA

E-mail address: b.bollobas@dpmms.cam.ac.uk

Department of Mathematics, Uppsala University, PO Box 480, SE-751 06 Uppsala, Sweden

E-mail address: svante.janson@math.uu.se

URL: http://www.math.uu.se/ svante/

Mathematical Institute, University of Oxford, 24-29 St Giles', Oxford OX1 3LB, UK

E-mail address: riordan@maths.ox.ac.uk 